\title{
Implementing Option Pricing Models When Asset Returns Are Predictable
}

\author{
ANDREW W. LO and JIANG WANG*
}

\begin{abstract}
The predictability of an asset's returns will affect the prices of options on that asset, even though predictability is typically induced by the drift, which does not enter the option pricing formula. For discretely-sampled data, predictability is linked to the parameters that do enter the option pricing formula. We construct an adjustment for predictability to the Black-Scholes formula and show that this adjustment can be important even for small levels of predictability, especially for longer maturity options. We propose several continuous-time linear diffusion processes that can capture broader forms of predictability, and provide numerical examples that illustrate their importance for pricing options.
\end{abstract}

THERE IS NOW A substantial body of evidence that documents the predictability of financial asset returns. ${ }^{1}$ Despite the lack of consensus as to the sources of such predictability - some attribute it to time-varying expected returns, perhaps due to changes in business conditions, while others argue that predictability is a symptom of inefficient markets or irrational investors - there is a growing consensus that predictability is a genuine feature of many financial asset returns.

In this article, we investigate the impact of asset return predictability on the prices of an asset's options. A comparison between the polar cases of perfect predictability (certainty) and perfect unpredictability (the random walk) suggests that predictability must have an effect on option prices, although what that effect might be is far from obvious. However, in the

\footnotetext{
* Both authors are from the Sloan School of Management, Massachusetts Institute of Technology. We thank Petr Adamek, Lars Hansen, John Heaton, Chi-fu Huang, Ravi Jagannathan, Barbara Jansen, René Stulz, and especially Bruce Grundy and the referee for helpful suggestions, and seminar participants at Boston University, Northwestern University, the Research Triangle Econometrics Workshop, the University of Texas at Austin, the University of Chicago, Washington University, the University of California at Los Angeles, the Wharton School, and Yale University for their comments. Financial support from the Laboratory for Financial Engineering is gratefully acknowledged. A portion of this research was conducted during the first author's tenure as an Alfred P. Sloan Research Fellow.

${ }^{1}$ See, for example, Bekaert and Hodrick (1992), Bessembinder and Chan (1992), Breen, Glosten, and Jagannathan (1989), Campbell and Ammer (1993), Campbell and Hamao (1992), Chan (1992), Chen (1991), Chen, Roll, and Ross (1986), Chopra, Lakonishok, and Ritter (1992), DeBondt and Thaler (1985), Engle, Lilien, and Robbins (1987), Fama and French (1988a, 1988b, 1990), Ferson (1989, 1990), Ferson, Foerster, and Keim (1993), Ferson and Harvey (1991a, 1991b), Ferson, Kandel, and Stambaugh (1987), Gibbons and Ferson (1985), Harvey (1989b), Jegadeesh (1990), Keim and Stambaugh (1986), King (1966), Lehmann (1990), Lo and MacKinlay (1988, 1990, 1992), and Poterba and Summers (1988).
} 
continuous-time no-arbitrage pricing framework of Black and Scholes (1973) and Merton (1973), and in the martingale pricing approach of Cox and Ross (1976) and Harrison and Kreps (1979), option pricing formulas are shown to be functionally independent of the drift of the price process. Since the drift is usually where predictability manifests itself-it is, after all, the conditional expectation of (instantaneous) returns - this seems to imply that predictability is irrelevant for option prices. ${ }^{2}$

The source of this apparent paradox lies in the attempt to link the properties of finite holding-period returns, such as predictability, to the properties of infinitesimal returns, such as the instantaneous volatility $\sigma$, without properly fixing the appropriate quantities. In particular, while it is true that changes in predictability arising from the drift cannot affect option prices under the Black-Scholes assumption that $\sigma$ is fixed, fixing $\sigma$ implies that the unconditional variance of finite holding-period returns will change as predictability changes. But since the unconditional variance of returns is usually fixed for any given set of data irrespective of predictability-for example, the historical annual standard deviation of the return on the S\&P 500 Index is 19.9 percent-fixing $\sigma$ and varying predictability can yield counterfactual implications for the data. ${ }^{3}$

The resolution of the paradox lies in the observation that if we fix the unconditional variance of the "true" (finite holding period) asset return process, i.e., the data, then as more predictability is introduced via the drift, the population value of the diffusion coefficient must change so as to keep the unconditional variance constant. Therefore, although the option pricing formula is unaffected by changes in predictability, option prices do change. In this respect, ignoring predictability in the drift is tantamount to committing a specification error that can lead to incorrect prices just as any other specification error can (e.g., Merton (1976b)).

But why should the unconditional variance be fixed? One answer is provided by the fact that the marginal distribution of asset returns is a more fundamental or primitive object than the joint distribution of asset returns and other economic variables. Therefore, a logical sequence of investigation is to first match the marginal distribution of returns, and then focus on the implications for the joint and conditional distributions. This is the approach typically taken in studies of the predictability of asset returns. When regres-

\footnotetext{
${ }^{2}$ Predictability can also manifest itself in the diffusion coefficient, in the form of stochastic volatility with dynamics that depend on predetermined economic factors. However, since predictability is more commonly modeled as part of the conditional mean, we shall focus solely on the drift.

${ }^{3}$ In fact, we argue more generally below that all of the unconditional moments of the marginal distribution of returns are "fixed," in the sense that empirical estimates of their values are readily obtained from the data, hence any economic or statistical model of predictability must be calibrated to these values to be of empirical relevance. But there is a compelling reason for focusing first on the unconditional variance of returns: any sensible comparative static analysis of predictability must keep fixed the unconditional variance of the variable to be predicted, since this is the benchmark against which the predictive power of a forecast is to be measured.
} 
sors are added to or subtracted from a forecasting equation, the conditional moments of returns change, affecting the joint distribution of returns and predictors, but the unconditional moments of the marginal distribution of returns, e.g., mean, variance, skewness, kurtosis, etc., remain the same as long as the data are fixed. ${ }^{4}$

Of course, when choosing among several competing specifications of the data, we hope to select the specification that matches most closely all of its properties, i.e., its finite-dimensional distributions. ${ }^{5}$ But since our most basic understanding of and intuition for the data comes from its marginal distribution, at the very least we shall require that any plausible specification must match the marginal distribution's unconditional moments. ${ }^{6}$ This is tantamount to fixing the mean, variance, skewness, etc. at the "true" values.

Alternatively, from a purely empirical standpoint, the unconditional sample moments of the data are fixed at a given point in time since we have only one historical realization of each asset return series. But the conditional moments of the data depend on the conditioning information, which changes as we learn more about the underlying economic structure of the data. The specification searches that we undertake can almost always be viewed as an attempt to fit a statistical model to these fixed sample moments.

Finally, yet another symptom of the link between predictability and option prices is the observation that implied volatilities will generally be biased estimates of the sample volatility of finite holding-period asset returns in the presence of predictability (see, for example, equation (24) below). The nature and magnitude of such biases depend on the nature and magnitude of predictability.

By fixing the unconditional moments of the data and specifying the form of predictability in asset returns, we show that changes in predictability affect the population value of the diffusion coefficient, and this in turn will affect option prices. The particular effect on option prices depends critically on how predictability is specified in the drift. For example, if the drift depends only on exogenous time-varying economic factors, then an increase in predictability unambiguously decreases option values. But if the drift also depends upon lagged prices, then an increase in predictability can either increase or decrease option values, depending on the particular specification of the drift.

\footnotetext{
${ }^{4}$ This is also the approach taken in the growing "calibration" literature begun by Mehra and Prescott (1985). More recent examples include Abel (1992), Cecchetti, Lam, and Mark (1993), Heaton and Lucas $(1992,1994)$, Kandel and Stambaugh $(1988,1990)$, and Weil (1989).

${ }^{5}$ Although the finite-dimensional distributions do not completely determine a continuous-time stochastic process, for our purposes they shall suffice. More rigorously, the concepts of separability and measurability must be introduced to complete the definition of continuous-time processes - see, for example, Doob (1953, Chapter II.2).

${ }^{6}$ For convenience, we shall refer to the unconditional moments of the marginal distribution of returns as simply the "unconditional moments." These moments are not to be confused with unconditional "co-moments," which are moments of the joint distribution of returns, not of the marginals.
} 
We derive explicit pricing formulas for options on assets with predictable returns, and show that even small amounts of predictability can have a large impact on option prices, especially for longer maturity options. For example, under the standard Black-Scholes assumption of a geometric random walk for stock prices, the price of a one-year at-the-money call option on a $\$ 40$ stock with a daily return volatility of 2 percent per day is $\$ 6.908$. However, under a trending Ornstein-Uhlenbeck (O-U) price process - which yields serially correlated returns - we show that a daily first-order autocorrelation coefficient of -0.20 and a daily return-volatility of 2 percent per day would yield an arbitrage-free option price of $\$ 7.660$, an increase of about 11 percent (see Section II.C and Table I). Of course, the particular adjustment to option prices depends on the specification of the drift, and we propose several specifications that can account for a broad variety of predictability in asset returns and illustrate the importance of these adjustments with several numerical examples.

In Section I we provide a brief review of the Black-Scholes option pricing model to clarify the role of the drift and to emphasize the distinction between the data-generating process and the "risk-neutralized" process for the underlying asset's price. The implications of this distinction for option prices are developed in Section II, where we present an adjustment for the volatility parameter $\sigma$ that accounts for the most parsimonious form of predictability: autocorrelation in asset returns. To account for more general forms of predictability, we propose two classes of linear diffusion processes in Sections III and IV, the bivariate and multivariate trending O-U processes, respectively. In Section V we show how the parameters of these predictable alternatives can be estimated with discretely sampled data by recasting them in statespace form and using the Kalman filter to obtain the likelihood function. We consider several extensions and qualifications in Section VI, and we conclude in Section VII.

\section{The Black-Scholes Option Pricing Formula and the Drift}

The fundamental insight of the option pricing models of Black and Scholes (1973) and Merton (1973) is the existence of a dynamic investment strategy involving the underlying asset and riskless bonds that replicates the option's payoff exactly. In particular, if the underlying asset's price process $P(t)$ satisfies the following stochastic differential equation:

$$
d \log P(t) \equiv d p(t)=\mu(\cdot) d t+\sigma d W,
$$

where $\sigma$ is the diffusion coefficient, $\mu(\cdot)$ the drift coefficient, $W(t)$ a standard Wiener process, and trading is frictionless and continuous, then the no-arbitrage condition yields the following restriction on the call option price $C$ :

$$
\frac{1}{2} \sigma^{2} P^{2} \frac{\partial^{2} C}{\partial P^{2}}+r P \frac{\partial C}{\partial P}+\frac{\partial C}{\partial t}=r C,
$$


where $r$ is the instantaneous risk-free rate of return. ${ }^{7}$ Given the two boundary conditions for the call option, $C(P(T), T)=\operatorname{Max}[P(T)-K, 0]$ and $C(0, t)$ $=0$, there exists a unique solution to the partial differential equation (2), the celebrated Black-Scholes formula:

$$
C_{B S}(P(t), t ; K, T, r, \sigma)=P(t) \Phi\left(d_{1}\right)-K e^{-r(T-t)} \Phi\left(d_{2}\right),
$$

where:

$$
d_{1} \equiv \frac{\log (P(t) / K)+\left(r+\frac{1}{2} \sigma^{2}\right)(T-t)}{\sigma \sqrt{T-t}}, \quad d_{2} \equiv d_{1}-\sigma \sqrt{T-t},
$$

and $\Phi(\cdot)$ is the standard normal cumulative distribution function.

Although it is well-known that the Black-Scholes formula does not depend on the drift $\mu(\cdot)$, it is rarely emphasized that the drift need not be a constant as in the case of geometric Brownian motion, but may be an arbitrary function of $P$ and other economic variables. ${ }^{8}$ This remarkable fact implies that the Black-Scholes formula is applicable to a wide variety of price processes, processes that exhibit complex patterns of predictability and dependence on other observed and unobserved economic factors (see, for example, the processes described in Sections III and IV below).

The second and more modern approach to pricing options is to construct an equivalent martingale measure, which is always possible if prices are set so that arbitrage opportunities do not exist. The martingale pricing method explicitly exploits the fact that the pricing equation is independent of the drift. Since the drift $\mu(\cdot)$ does not enter into the pricing equation (2), for purposes of pricing options it may be set to any arbitrary function without loss of generality (subject to some regularity conditions). In particular, under the equivalent martingale measure in which all asset prices follow martingales, the option's price is simply the present discounted value of its expected payoff at maturity, where the expectation is computed with respect to the risk-neutralized process $P^{*}(t)$ where:

$$
d \log P^{*}(t)=\left(r-\frac{\sigma^{2}}{2}\right) d t+\sigma d W .
$$

Although the risk-neutralized process is not empirically observable, it is nevertheless an extremely convenient specification for evaluating the price of an option on the stock with a data-generating process given by $P(t)$.

The two approaches to pricing derivative assets show that as long as the diffusion coefficient for the log-price process is a known constant $\sigma$, then the

\footnotetext{
${ }^{7}$ That $C$ is a function only of $P$ and $t$, twice differentiable in $P$ and once differentiable in $t$ are properties that can be derived from the replicating strategy and need not be assumed a priori. See Merton (1973) for further details.

${ }^{8}$ This was first observed by Merton (1973), and it is also explicitly acknowledged by Jagannathan (1984) and Grundy (1991), but is far too often overlooked in textbook expositions of the Black-Scholes formula. Of course, $\mu(\cdot)$ must still satisfy some regularity conditions to ensure the existence of a solution to the stochastic differential equation (1).
} 
Black-Scholes formula yields the correct option price regardless of the specification and arguments of the drift. ${ }^{9}$ But the fact that the drift plays no role in determining a derivative's pricing formula belies its importance in the formula's implementation. Because the risk-neutral distribution and the true distribution of the data-generating process are linked, predictability can and generally does have an influence on the pricing of derivative assets, despite the fact that only the parameters of the risk-neutral distribution appear in derivative pricing formulas. As we show in the next sections, when we change the true distribution of the data-generating process, e.g., change predictability, the associated risk-neutral distribution also changes, and these changes will generally affect option prices.

\section{Option Prices and Predictability}

Although the same symbol $\sigma$ is used in both the risk-neutralized process $P^{*}$ and the data-generating process $P$, both the theoretical value and the empirical estimate of $\sigma$ are determined solely by the data-generating process, not by the risk-neutralized process, and both will be affected by the functional form of the drift. Predictability in the drift can be safely ignored when deriving the option pricing formula, but we shall argue below that it must be addressed explicitly for any given data-generating process.

In Section II. $A$, we consider the most parsimonious form of predictability-autocorrelated asset returns-and show how it affects $\sigma$ directly in the specific case of a trending O-U process for log-prices. We also provide a simple adjustment to the Black-Scholes formula that can account for it. More general and empirically plausible log-price processes, with considerably more flexible forms of predictability, are presented in Sections III and IV.

\section{A. The Trending $O-U$ Process}

In distinguishing between the risk-neutral and true distributions of an option's underlying asset return process, Grundy (1991, p. 1049) observes that the Black-Scholes formula still holds for an O-U log-price process, and we shall begin with a slight generalization of his example to illustrate the link between predictablity and option prices. Specifically, let the log-price process $p(t)$ satisfy the following stochastic differential equation:

$$
d p(t)=(-\gamma(p(t)-\mu t)+\mu) d t+\sigma d W
$$

where $\gamma \geq 0, p(0)=p_{0}$, and $t \in[0, \infty)$. Unlike the original Black-Scholes model, which assumes that log-prices follow an arithmetic random walk with

\footnotetext{
${ }^{9}$ More generally, it may be shown that for any derivative asset that can be priced purely by arbitrage, and where the underlying asset's log-price dynamics is described by an Itô diffusion with constant diffusion coefficient, the derivative pricing formula is functionally independent of the drift and is determined purely by the diffusion coefficient and the contract specifications of the derivative asset.
} 
independently and identically distributed Gaussian increments, this log-price process is the sum of a zero-mean stationary autoregressive Gaussian process - an O-U process - and a deterministic linear trend, so we call this the "trending O-U" process. Rewriting equation (6) as:

$$
d(p(t)-\mu t)=-\gamma(p(t)-\mu t) d t+\sigma d W
$$

shows that when $p(t)$ deviates from its trend $\mu t$, it is pulled back at a rate proportional to its deviation where $\gamma$ is the "speed of adjustment." ${ }^{0}$ For notational convenience, we shall work with the detrended log-price process $q(t)$ for the remainder of this article, where $q(t) \equiv p(t)-\mu t$. From equation (7), we have:

$$
d q(t)=-\gamma q(t) d t+\sigma d W
$$

and $q(0)=q_{0}=p_{0}$, which has the following explicit solution:

$$
q(t)=e^{-\gamma t} q_{0}+\sigma \int_{0}^{t} e^{-\gamma(t-s)} d W(s) .
$$

To develop further intuition for the behavior of the trending O-U process, the Appendix reports the moments of finite holding-period returns associated with the detrended log-price process in equation (8).

Unlike the arithmetic Brownian motion or random walk, which is nonstationary and often said to be "difference-stationary" or a "stochastic trend," the trending O-U process is said to be "trend-stationary" since its deviations from trend follow a stationary process. An implication of trend-stationarity is that the unconditional variance of $\tau$-period returns has a finite limit as $\tau$ increases without bound-in this case $\sigma^{2} / \gamma$-in contrast to the case of a random walk in which the unconditional variance increases linearly with $\tau$. This difference between the trending $\mathrm{O}-\mathrm{U}$ process and the random walk also exists for the conditional variance of continuously compounded returns $r_{\tau}(t)$ $\equiv p(t)-p(t-\tau)$. In fact, for the trending O-U process we have

$$
\operatorname{Var}\left[r_{\tau}(t) \mid p(t)\right]=\frac{\sigma^{2}}{2 \gamma}\left[1-e^{-2 \gamma \tau}\right], \quad \gamma>0
$$

which does not equal $\sigma^{2} \tau$ as in the case of the random walk. The conditional variance of $\tau$-period returns also has a finite limit (when $\gamma>0$ ) as $\tau \rightarrow \infty .^{11}$

Note that the first-order autocorrelation of the trending O-U increments is always less than or equal to zero, bounded below by $-\frac{1}{2}$, and approaches $-\frac{1}{2}$ as $\tau$ increases without bound (see equation (A4) in the Appendix). These, and other aspects of the trending $\mathrm{O}-\mathrm{U}$ process, will prove to be serious restrictions for many empirical applications and will motivate the alternative processes introduced in Sections III and IV, which have more flexible autocorrelation

${ }^{10}$ Note that $\gamma>0$ ensures the stationarity of $p(t)$.

${ }^{11}$ Observe that the conditional variance (equation (10)) is conditioned on only the current price $p(t)$. Of course, more general conditioning information can be used, with potentially different implications. We discuss this further in Section III. $B$. 
functions..$^{12}$ However, as an illustration of the impact of serial correlation on option prices the trending $\mathrm{O}-\mathrm{U}$ process is ideal.

\section{B. Relating Unconditional Moments to Parameters}

Despite the differences between the trending O-U process and an arithmetic Brownian motion, Grundy (1991) points out that both data-generating processes yield the same risk-neutralized price process (equation (5)) hence the Black-Scholes formula still applies to options on stocks with log-price dynamics given by equation (6). This may seem paradoxical, especially since the Black-Scholes formula is independent of the parameter $\gamma$, which determines the degree of autocorrelation in returns. After all, autocorrelation is a simple form of predictability, and we have argued in the introduction that predictability should have some impact on option prices.

The paradox is readily resolved by observing that the two data-generating processes, equations (1) and (6), must fit the same price data - they are, after all, two competing specifications of a single price process, the "true" data-generating process. Therefore, in the presence of autocorrelation, equation (6), the numerical value for the Black-Scholes input $\sigma$ will be different than in the case of no autocorrelation, equation (1).

To be concrete, denote by $\bar{r}_{\tau}, s^{2}\left(r_{\tau}\right)$, and $\rho_{\tau}(1)$ the unconditional mean, variance, and first-order autocorrelation of $r_{\tau}(t)$, respectively, which may be defined without reference to any particular data-generating process. ${ }^{13}$ Moreover, the numerical values of these quantities may also be fixed without reference to any particular data-generating process. All competing specifications for the true data-generating process must match these moments at the very least to be plausible descriptions of that data (of course, the best specification is one that matches all the moments, in which case the true data-generating process will have been discovered). For the arithmetic Brownian motion, this implies that the parameters $\left(\mu, \sigma^{2}\right)$ must satisfy the following relations:

$$
\begin{aligned}
\bar{r}_{\tau} & =\mu \tau \\
s^{2}\left(r_{\tau}\right) & =\sigma^{2} \tau \\
\rho_{\tau}(1) & =0 .
\end{aligned}
$$

${ }^{12}$ While trend-stationary processes are often simpler to estimate, they have been criticized as unrealistic models of financial asset prices since they do not accord well with the common intuition that longer-horizon asset returns exhibit more risk or that price forecasts exhibit more uncertainty as the forecast horizon grows. However, if the source of such intuition is empirical observation, it may be consistent with trend-stationarity, since it is now well known that for any finite set of data trend-stationarity and difference-stationarity are virtually indistinguishable (e.g., Campbell and Perron (1991) and the many other "unit root" papers cited in their references). Nevertheless, in Section IV we shall provide a generalization of the trending O-U process that contains stochastic trends, in which case the variance of returns will increase without bound with the holding period $\tau$.

${ }^{13}$ Of course, it must be assumed that the moments exist. However, even if they do not, a similar but more involved argument may be based on location, scale, and association parameters. 
From equation (12), we obtain the well-known result that the Black-Scholes input $\sigma^{2}$ may be estimated by the sample variance of continuously compounded returns $r_{\tau}$. However, in the case of the trending O-U process, the parameters $\left(\mu, \gamma, \sigma^{2}\right)$ must satisfy:

$$
\begin{aligned}
\bar{r}_{\tau} & =\mu \tau \\
s^{2}\left(r_{\tau}\right) & =\frac{\sigma^{2}}{\gamma}\left[1-e^{-\gamma \tau}\right], \quad r \geq 0 \\
\rho_{\tau}(1) & =-\frac{1}{2}\left[1-e^{-\gamma \tau}\right] .
\end{aligned}
$$

Observe that these relations must hold for the theoretical or population values of the parameters if the trending $\mathrm{O}-\mathrm{U}$ process is to be a plausible description of the data-generating process. Moreover, while equations (14) to (16) involve population values of the parameters, they also have implications for estimation. In particular, under the trending O-U specification, the sample variance of continuously-compounded returns is clearly not an appropriate estimator for $\sigma^{2}$.

Holding the unconditional variance of returns fixed, the particular value of $\sigma^{2}$ now depends on $\gamma$. Solving equations (15) and (16) for $\gamma$ and $\sigma^{2}$ yields:

$$
\begin{aligned}
\gamma & =-\frac{1}{\tau} \log \left(1+2 \rho_{\tau}(1)\right) \\
\sigma^{2} & =s^{2}\left(r_{\tau}\right) \gamma\left(1-e^{-\gamma \tau}\right)^{-1}=\frac{s^{2}\left(r_{\tau}\right)}{\tau} \cdot\left[\gamma \tau\left(1-e^{-\gamma \tau}\right)^{-1}\right] .
\end{aligned}
$$

which shows the dependence of $\sigma^{2}$ on $\gamma$ explicitly.

In the second equation of expression (18), $\sigma^{2}$ has been re-expressed as the product of two terms: the first is the standard Black-Scholes input under the assumption that arithmetic Brownian motion is the data-generating process, and the second is an adjustment factor required by the trending O-U specification. Since this adjustment factor is an increasing function of $\gamma$, as returns become more highly (negatively) autocorrelated, options on the stock will become more valuable ceteris paribus. Specifically, substituting equation (17) into equation (18) and simplifying yields $\sigma^{2}$ as an explicit function of $\rho_{\tau}(1)$ :

$$
\sigma^{2}=\frac{s^{2}\left(r_{\tau}\right)}{\tau} \cdot \frac{\log \left(1+2 \rho_{\tau}(1)\right)}{2 \rho_{\tau}(1)}, \quad \rho_{\tau}(1) \in\left(-\frac{1}{2}, 0\right] .
$$

where the restriction that $\rho_{\tau}(1) \in\left(-\frac{1}{2}, 0\right]$ is equivalent to the restriction that $\gamma \geq 0$.

More generally, suppose that returns of one holding period $\tau_{1}$ are used to obtain the unconditional variance $s^{2}\left(r_{\tau_{1}}\right)$, and returns of another holding period $\tau_{2}$ are used to obtain the first-order autocorrelation coefficient $\rho_{\tau_{2}}(1)$. Since the data-generating process is defined in continuous time, this poses no problems for deriving the restrictions on the parameters $\left(\mu, \gamma, \sigma^{2}\right)$, and 
manipulating those restrictions yields the following version of equation (19):

$$
\begin{aligned}
\sigma^{2} & =\frac{s^{2}\left(r_{\tau_{1}}\right)}{\tau_{1}} \cdot \frac{\tau_{1}}{\tau_{2}} \cdot \frac{\log \left(1+2 \rho_{\tau_{2}}(1)\right)}{\left[1+2 \rho_{\tau_{2}}(1)\right]^{\tau_{1} / \tau_{2}}-1} \\
& =\frac{s^{2}\left(r_{\tau_{1}}\right)}{\tau_{1}} \cdot \Delta\left(\tau_{1}, \tau_{2}, \rho_{\tau_{2}}(1)\right) \\
\Delta\left(\tau_{1}, \tau_{2}, \rho_{\tau_{2}}(1)\right) \equiv & \frac{\tau_{1}}{\tau_{2}} \cdot \frac{\log \left(1+2 \rho_{\tau_{2}}(1)\right)}{\left[1+2 \rho_{\tau_{2}}(1)\right]^{\tau_{1} / \tau_{2}}-1}, \quad \rho_{\tau_{2}}(1) \in\left(-\frac{1}{2}, 0\right] .
\end{aligned}
$$

Without loss of generality and as a convenient normalization, let $\tau_{1}=1$ and $\tau_{2}=\tau$, so that the first-order autocorrelation coefficient $\rho_{\tau}(1)$ is defined over the holding period $\tau$, which in turn is measured in units of the holding period used to measure the unconditional variance of returns $s^{2}\left(r_{1}\right)$, thus:

$$
\sigma^{2}=\frac{s^{2}\left(r_{1}\right)}{\tau} \cdot \frac{\log \left(1+2 \rho_{\tau}(1)\right)}{\left[1+2 \rho_{\tau}(1)\right]^{1 / \tau}-1}, \quad \rho_{\tau}(1) \in\left(-\frac{1}{2}, 0\right] .
$$

This expression provides a simple adjustment for the Black-Scholes input $\sigma^{2}$ using the unconditional variance $s^{2}\left(r_{1}\right)$ of returns sampled at unit intervals, and the first-order autocorrelation $\rho_{\tau}(1)$ of returns sampled at $\tau$-intervals.

Returning to the simpler relation, equation (19), between $\sigma^{2}$ and the first-order autocorrelation coefficient, and holding fixed the unconditional variance of returns $s^{2}\left(r_{\tau}\right)$, observe that the value of $\sigma^{2}$ increases without bound as the absolute value of the autocorrelation increases from 0 to $\frac{1}{2} \cdot{ }^{14}$ This implies that a specification error in the dynamics of $p(t)$ can have dramatic consequences for pricing options. We shall quantify the magnitudes of such consequences in Sections II. $C$ and III. $B$ below.

\section{Implications for Option Prices}

Expression (19) provides the necessary input to the Black-Scholes formula for pricing options on an asset with the trending O-U dynamics. In particular, if the unconditional variance of daily returns is $s^{2}\left(r_{1}\right)$, and if the first-order autocorrelation of $\tau$-period returns is $\rho_{\tau}(1)$, then the price of a call option is

\footnotetext{
${ }^{14}$ We focus on the absolute value of the autocorrelation to avoid confusion in making comparisons between results for negatively autocorrelated and positively autocorrelated asset returns. For example, whereas in this case an increase in the absolute value of autocorrelation increases the option's value, in Section III. $B$ we provide an example of a positively autocorrelated asset return process for which an increase in autocorrelation decreases the option's value. These two cases are indeed polar opposites, and for important reasons. But without focusing on the absolute value of the autocorrelation, they seem to be in agreement: in both cases, the option price is a decreasing function of the algebraic value of the autocorrelation.
} 
given by:

$$
C_{\mathrm{OU}}=C_{\mathrm{BS}}(P(t), t ; K, T, r, \sigma)
$$

but where $\sigma$ is given by:

$$
\sigma^{2} \equiv \frac{s^{2}\left(r_{1}\right)}{\tau} \cdot \frac{\log \left(1+2 \rho_{\tau}(1)\right)}{\left[1+2 \rho_{\tau}(1)\right]^{1 / \tau}-1}, \quad \rho_{\tau}(1) \in\left(-\frac{1}{2}, 0\right] .
$$

This is simply the Black-Scholes formula with an adjusted volatility input, adjusted to account for the negative autocorrelation in the trending O-U process. ${ }^{15}$ In particular, the adjustment factor multiplying $s^{2}\left(r_{1}\right) / \tau$ in equation (25) is easily tabulated (see Table V and the discussion in Section VI.B), hence in practice it is a simple matter to adjust the Black-Scholes formula for negative autocorrelation of the form in equation (16): multiply the usual variance estimator $s^{2}\left(r_{1}\right) / \tau$ by the appropriate factor from Table $\mathrm{V}$ and use this as $\sigma^{2}$ in the Black-Scholes formula.

Note that for all values of $\rho_{\tau}(1)$ in $\left(-\frac{1}{2}, 0\right]$, the factor multiplying $s^{2}\left(r_{1}\right) / \tau$ in equation (25) is greater than or equal to one, and increasing in the absolute value of the first-order autocorrelation coefficient. This implies that option values under the trending O-U specification are always greater than or equal to option values under the standard Black-Scholes specification, and that option values are an increasing function of the absolute value of the first-order autocorrelation coefficient. These are purely features of the trending O-U process and do not generalize to other specifications of the drift, as we shall see below.

To gauge the empirical relevance of this adjustment for autocorrelation, Tables I to III report a comparison of Black-Scholes prices under arithmetic Brownian motion and under the trending O-U process for various holding periods, strike prices, and autocorrelations for a hypothetical $\$ 40$ stock. Table I reports option prices for values of daily autocorrelations from -5 to -45 percent, and Tables II and III report prices for weekly and monthly autocorrelations of the same numerical values. For all three tables, the unconditional standard deviation of daily returns is held fixed at 2 percent per day. The Black-Scholes price is calculated according to equation (3), setting $\sigma$ equal to the unconditional standard deviation. The trending $\mathrm{O}-\mathrm{U}$ prices are calculated by solving equations (15) and (16) for $\sigma$ given $\tau$ and the return autocorrelations $\rho_{\tau}(1)$ of $-0.05,-0.10,-0.20,-0.30,-0.40$, and -0.45 , and using these values of $\sigma$ in the Black-Scholes formula (equation (3)). In Table I, $\tau=1$; in Tables II and III, $\tau=7$ and 364/12, respectively.

Panel A of Table I shows that even extreme autocorrelation in daily returns does not affect short-maturity in-the-money call option prices very much. For

\footnotetext{
${ }^{15}$ Since $s^{2}\left(r_{1}\right)$ is the unconditional variance of daily returns, observe that $\sigma^{2}$ is also measured in days, as is the time-to-maturity $T-t$ and the interest rate $r$.
} 
Table I

\section{Option Prices Under the Trending Ornstein-Uhlenbeck Process (Daily Frequency)}

Comparison of Black-Scholes call option prices on a hypothetical $\$ 40$ stock under an arithmetic Brownian motion versus a trending Ornstein-Uhlenbeck $(\mathrm{O}-\mathrm{U})$ process for log-prices, assuming a standard deviation of 2 percent for daily continuously-compounded returns, and a daily continuously-compounded riskfree rate of $\log (1.05) / 364$. As autocorrelation becomes larger in absolute value, option prices increase.

\begin{tabular}{|c|c|c|c|c|c|c|c|}
\hline \multirow{2}{*}{$\begin{array}{l}\text { Strike } \\
\text { Price }\end{array}$} & \multirow{2}{*}{$\begin{array}{c}\text { Black-Scholes } \\
\text { Price }\end{array}$} & \multicolumn{6}{|c|}{ Trending O-U Price, with Daily $\rho_{\tau}(1)=$} \\
\hline & & -0.05 & -0.10 & -0.20 & -0.30 & -0.40 & -0.45 \\
\hline \multicolumn{8}{|c|}{ Panel A. Time-to-Maturity $T-t=7$ Days } \\
\hline 30 & 10.028 & 10.028 & 10.028 & 10.028 & 10.028 & 10.028 & 10.028 \\
\hline 35 & 5.036 & 5.037 & 5.038 & 5.042 & 5.051 & 5.074 & 5.108 \\
\hline 40 & 0.863 & 0.885 & 0.910 & 0.973 & 1.062 & 1.216 & 1.368 \\
\hline 45 & 0.011 & 0.013 & 0.016 & 0.024 & 0.041 & 0.082 & 0.137 \\
\hline 50 & 0.000 & 0.000 & 0.000 & 0.000 & 0.000 & 0.001 & 0.005 \\
\hline \multicolumn{8}{|c|}{ Panel B. Time-to-Maturity $T-t=91$ Days } \\
\hline 30 & 10.526 & 10.547 & 10.572 & 10.640 & 10.754 & 10.989 & 11.262 \\
\hline 35 & 6.331 & 6.387 & 6.451 & 6.614 & 6.855 & 7.289 & 7.735 \\
\hline 40 & 3.270 & 3.350 & 3.439 & 3.661 & 3.978 & 4.526 & 5.068 \\
\hline 45 & 1.459 & 1.532 & 1.615 & 1.822 & 2.124 & 2.657 & 3.195 \\
\hline 50 & 0.574 & 0.623 & 0.680 & 0.829 & 1.058 & 1.491 & 1.954 \\
\hline \multicolumn{8}{|c|}{ Panel C. Time-to-Maturity $T-t=182$ Days } \\
\hline 30 & 11.285 & 11.336 & 11.394 & 11.548 & 11.786 & 12.238 & 12.725 \\
\hline 35 & 7.558 & 7.646 & 7.746 & 7.998 & 8.365 & 9.014 & 9.668 \\
\hline 40 & 4.740 & 4.851 & 4.976 & 5.286 & 5.728 & 6.491 & 7.244 \\
\hline 45 & 2.810 & 2.922 & 3.048 & 3.361 & 3.812 & 4.595 & 5.375 \\
\hline 50 & 1.592 & 1.687 & 1.797 & 2.073 & 2.482 & 3.214 & 3.963 \\
\hline \multicolumn{8}{|c|}{ Panel D. Time-to-Maturity $T-t=273$ Days } \\
\hline 30 & 12.040 & 12.113 & 12.198 & 12.415 & 12.745 & 13.352 & 13.989 \\
\hline 35 & 8.587 & 8.698 & 8.824 & 9.139 & 9.596 & 10.396 & 11.199 \\
\hline 40 & 5.905 & 6.039 & 6.191 & 6.565 & 7.099 & 8.019 & 8.925 \\
\hline 45 & 3.943 & 4.082 & 4.239 & 4.627 & 5.185 & 6.147 & 7.098 \\
\hline 50 & 2.573 & 2.702 & 2.849 & 3.217 & 3.753 & 4.695 & 5.642 \\
\hline \multicolumn{8}{|c|}{ Panel E. Time-to-Maturity $T-t=364$ Days } \\
\hline 30 & 12.753 & 12.845 & 12.950 & 13.218 & 13.620 & 14.349 & 15.102 \\
\hline 35 & 9.493 & 9.622 & 9.769 & 10.133 & 10.661 & 11.582 & 12.501 \\
\hline 40 & 6.908 & 7.061 & 7.234 & 7.660 & 8.269 & 9.315 & 10.343 \\
\hline 45 & 4.941 & 5.102 & 5.283 & 5.732 & 6.374 & 7.478 & 8.566 \\
\hline 50 & 3.489 & 3.645 & 3.821 & 4.261 & 4.896 & 6.003 & 7.106 \\
\hline
\end{tabular}

example, a daily autocorrelation of -45 percent has no impact on the $\$ 30$ 7-day call; the price under the trending $\mathrm{O}-\mathrm{U}$ process is identical to the standard Black-Scholes price of $\$ 10.028$. But even for such a short maturity, differences become more pronounced as the strike price increases; the at-the- 
Table II

\section{Option Prices Under the Trending Ornstein-Uhlenbeck Process (Weekly Frequency)}

Comparison of theoretical call option prices on a hypothetical $\$ 40$ stock under an arithmetic Brownian motion versus a trending Ornstein-Uhlenbeck $(\mathrm{O}-\mathrm{U})$ process for log-prices, assuming a standard deviation of 2 percent for daily continuously-compounded returns, and a daily continuously-compounded riskfree rate of $\log (1.05) / 364$. As autocorrelation becomes larger in absolute value, option prices increase.

\begin{tabular}{|c|c|c|c|c|c|c|c|}
\hline \multirow{2}{*}{$\begin{array}{l}\text { Strike } \\
\text { Price }\end{array}$} & \multirow{2}{*}{$\begin{array}{c}\text { Black-Scholes } \\
\text { Price }\end{array}$} & \multicolumn{6}{|c|}{ Trending O-U Price, with Weekly $\rho_{\tau}(1)=$} \\
\hline & & -0.05 & -0.10 & -0.20 & -0.30 & -0.40 & -0.45 \\
\hline \multicolumn{8}{|c|}{ Panel A. Time-to Maturity $T-t=7$ Days } \\
\hline 30 & 10.028 & 10.028 & 10.028 & 10.028 & 10.028 & 10.028 & 10.028 \\
\hline 35 & 5.036 & 5.036 & 5.037 & 5.037 & 5.037 & 5.038 & 5.040 \\
\hline 40 & 0.863 & 0.866 & 0.870 & 0.878 & 0.891 & 0.912 & 0.933 \\
\hline 45 & 0.011 & 0.011 & 0.011 & 0.012 & 0.013 & 0.016 & 0.019 \\
\hline 50 & 0.000 & 0.000 & 0.000 & 0.000 & 0.000 & 0.000 & 0.000 \\
\hline \multicolumn{8}{|c|}{ Panel B. Time-to-Maturity $T-t=91$ Days } \\
\hline 30 & 10.526 & 10.529 & 10.532 & 10.540 & 10.552 & 10.573 & 10.596 \\
\hline 35 & 6.331 & 6.338 & 6.347 & 6.369 & 6.400 & 6.455 & 6.510 \\
\hline 40 & 3.270 & 3.281 & 3.294 & 3.325 & 3.369 & 3.444 & 3.520 \\
\hline 45 & 1.459 & 1.470 & 1.481 & 1.510 & 1.550 & 1.620 & 1.690 \\
\hline 50 & 0.574 & 0.581 & 0.588 & 0.608 & 0.635 & 0.683 & 0.733 \\
\hline \multicolumn{8}{|c|}{ Panel C. Time-to-Maturity $T-t=182$ Days } \\
\hline 30 & 11.285 & 11.292 & 11.300 & 11.320 & 11.348 & 11.398 & 11.449 \\
\hline 35 & 7.558 & 7.570 & 7.584 & 7.619 & 7.668 & 7.752 & 7.838 \\
\hline 40 & 4.740 & 4.756 & 4.774 & 4.817 & 4.878 & 4.983 & 5.089 \\
\hline 45 & 2.810 & 2.826 & 2.844 & 2.887 & 2.949 & 3.055 & 3.162 \\
\hline 50 & 1.592 & 1.605 & 1.620 & 1.658 & 1.711 & 1.803 & 1.897 \\
\hline \multicolumn{8}{|c|}{ Panel D. Time-to-Maturity $T-t=273$ Days } \\
\hline 30 & 12.040 & 12.050 & 12.062 & 12.090 & 12.131 & 12.203 & 12.276 \\
\hline 35 & 8.587 & 8.603 & 8.621 & 8.664 & 8.726 & 8.832 & 8.939 \\
\hline 40 & 5.905 & 5.924 & 5.945 & 5.998 & 6.072 & 6.199 & 6.327 \\
\hline 45 & 3.943 & 3.962 & 3.984 & 4.039 & 4.116 & 4.248 & 4.381 \\
\hline 50 & 2.573 & 2.591 & 2.612 & 2.662 & 2.734 & 2.858 & 2.983 \\
\hline \multicolumn{8}{|c|}{ Panel E. Time-to-Maturity $T-t=364$ Days } \\
\hline 30 & 12.753 & 12.766 & 12.781 & 12.816 & 12.867 & 12.956 & 13.047 \\
\hline 35 & 9.493 & 9.512 & 9.532 & 9.582 & 9.654 & 9.777 & 9.902 \\
\hline 40 & 6.908 & 6.930 & 6.954 & 7.014 & 7.099 & 7.244 & 7.390 \\
\hline 45 & 4.941 & 4.964 & 4.989 & 5.052 & 5.141 & 5.294 & 5.448 \\
\hline 50 & 3.489 & 3.511 & 3.536 & 3.597 & 3.683 & 3.832 & 3.982 \\
\hline
\end{tabular}

money call is worth $\$ 0.863$ in the absence of autocorrelation but increases to $\$ 1.368$ with an autocorrelation of -45 percent.

As the time to maturity increases, the remaining panels of Table I show that the impact of autocorrelation also increases. With a -10 percent daily 


\section{Table III}

\section{Option Prices Under the Trending Ornstein-Uhlenbeck Process (Monthly Frequency)}

Comparison of theoretical call option prices on a hypothetical $\$ 40$ stock under an arithmetic Brownian motion versus a trending Ornstein-Uhlenbeck $(\mathrm{O}-\mathrm{U})$ process for log-prices, assuming a standard deviation of 2 percent for daily continuously-compounded returns, and a daily continuously-compounded riskfree rate of $\log (1.05) / 364$. As autocorrelation becomes larger in absolute value, option prices increase.

\begin{tabular}{|c|c|c|c|c|c|c|c|}
\hline \multirow{2}{*}{$\begin{array}{l}\text { Strike } \\
\text { Price }\end{array}$} & \multirow{2}{*}{$\begin{array}{c}\text { Black-Scholes } \\
\text { Price }\end{array}$} & \multicolumn{6}{|c|}{ Trending O-U Price, with Monthly $\rho_{\tau}(1)=$} \\
\hline & & -0.05 & -0.10 & -0.20 & -0.30 & -0.40 & -0.45 \\
\hline \multicolumn{8}{|c|}{ Panel A. Time-to-Maturity $T-t=7$ Days } \\
\hline 30 & 10.028 & 10.028 & 10.028 & 10.028 & 10.028 & 10.028 & 10.028 \\
\hline 35 & 5.036 & 5.036 & 5.036 & 5.036 & 5.037 & 5.037 & 5.037 \\
\hline 40 & 0.863 & 0.864 & 0.864 & 0.866 & 0.869 & 0.874 & 0.879 \\
\hline 45 & 0.011 & 0.011 & 0.011 & 0.011 & 0.011 & 0.012 & 0.012 \\
\hline 50 & 0.000 & 0.000 & 0.000 & 0.000 & 0.000 & 0.000 & 0.000 \\
\hline \multicolumn{8}{|c|}{ Panel B. Time-to-Maturity $T-t=91$ Days } \\
\hline 30 & 10.526 & 10.527 & 10.528 & 10.529 & 10.532 & 10.537 & 10.541 \\
\hline 35 & 6.331 & 6.332 & 6.334 & 6.339 & 6.346 & 6.359 & 6.371 \\
\hline 40 & 3.270 & 3.273 & 3.276 & 3.283 & 3.293 & 3.310 & 3.327 \\
\hline 45 & 1.459 & 1.462 & 1.464 & 1.471 & 1.480 & 1.496 & 1.512 \\
\hline 50 & 0.574 & 0.575 & 0.577 & 0.581 & 0.588 & 0.598 & 0.609 \\
\hline \multicolumn{8}{|c|}{ Panel C. Time-to-Maturity $T-t=182$ Days } \\
\hline 30 & 11.285 & 11.287 & 11.289 & 11.293 & 11.300 & 11.310 & 11.321 \\
\hline 35 & 7.558 & 7.561 & 7.564 & 7.572 & 7.583 & 7.602 & 7.621 \\
\hline 40 & 4.740 & 4.744 & 4.748 & 4.758 & 4.772 & 4.796 & 4.820 \\
\hline 45 & 2.810 & 2.814 & 2.818 & 2.828 & 2.842 & 2.866 & 2.890 \\
\hline 50 & 1.592 & 1.595 & 1.598 & 1.607 & 1.619 & 1.640 & 1.660 \\
\hline \multicolumn{8}{|c|}{ Panel D. Time-to-Maturity $T-t=273$ Days } \\
\hline 30 & 12.040 & 12.042 & 12.045 & 12.051 & 12.061 & 12.076 & 12.092 \\
\hline 35 & 8.587 & 8.591 & 8.595 & 8.605 & 8.619 & 8.643 & 8.667 \\
\hline 40 & 5.905 & 5.909 & 5.914 & 5.926 & 5.943 & 5.972 & 6.002 \\
\hline 45 & 3.943 & 3.947 & 3.952 & 3.965 & 3.982 & 4.012 & 4.043 \\
\hline 50 & 2.573 & 2.577 & 2.582 & 2.594 & 2.610 & 2.638 & 2.666 \\
\hline \multicolumn{8}{|c|}{ Panel E. Time-to-Maturity $T-t=364$ Days } \\
\hline 30 & 12.753 & 12.756 & 12.759 & 12.768 & 12.779 & 12.799 & 12.819 \\
\hline 35 & 9.493 & 9.497 & 9.502 & 9.514 & 9.530 & 9.558 & 9.586 \\
\hline 40 & 6.908 & 6.913 & 6.919 & 6.933 & 6.952 & 6.985 & 7.018 \\
\hline 45 & 4.941 & 4.946 & 4.952 & 4.966 & 4.987 & 5.022 & 5.057 \\
\hline 50 & 3.489 & 3.494 & 3.500 & 3.514 & 3.534 & 3.567 & 3.601 \\
\hline
\end{tabular}

autocorrelation, an at-the-money 1-year call is $\$ 7.234$, and rises to $\$ 10.343$ with a daily autocorrelation of -45 percent, compared to the standard Black-Scholes price of $\$ 6.908$. This pattern is not surprising, given the autocorrelation (equation (16)) of the trending O-U process, since longer- 
horizon returns are more highly negatively autocorrelated and, therefore, depart more severely from the standard Black-Scholes paradigm than shorter-horizon returns.

More formally, since the Black-Scholes formula applies to both arithmetic Brownian motion and the trending O-U process, the impact of a specification error in the drift can be related to the sensitivity of the Black-Scholes formula to changes in volatility $\sigma$. This sensitivity is measured by the derivative of the call price with respect to $\sigma$, and is often called the option's "vega" $\partial C / \partial \sigma=P(t) \sqrt{T-t} \Phi^{\prime}\left(d_{1}\right)$, where $d_{1}$ is defined in equation (4). From this expression, we see that the prices of shorter-maturity options are less sensitive to changes in $\sigma$, while the prices of longer-maturity options are more sensitive.

This is also apparent in the patterns of Tables II and III, which are similar to those in Table I but much less striking since the same numerical values of $\rho_{\tau}(1)$ are now assumed to hold for weekly and monthly returns, respectively. As Tables I to III show, the impact of a -45 percent autocorrelation in monthly returns is considerably less than the same autocorrelation in daily returns.

In contrast to Table I, where an at-the-money 1-year call increases from $\$ 6.908$ to $\$ 10.343$ as the autocorrelation decreases from 0 to -45 percent, in Table III the same option increases from $\$ 6.908$ to only $\$ 7.018$. We shall see in Table V of Section VI. $B$ that this is a symptom of all diffusion processes, since the increments of any diffusion process becomes less autocorrelated as the differencing interval declines. In particular, Table $\mathrm{V}$ will show that the impact of a -45 percent autocorrelation in monthly returns is considerably less than the same autocorrelation in daily returns. Indeed, from equation (16), a -45 percent autocorrelation in monthly returns implies an autocorrelation of only -0.97 percent in daily returns. Therefore, the importance of autocorrelation for option prices hinges critically on the degree of autocorrelation for a given return horizon $\tau$ and, of course, on the data-generating process that determines how rapidly this autocorrelation decays with $\tau$. For this reason, in the next section we introduce several new stochastic processes that are capable of matching more complex patterns of autocorrelation and predictability than the trending $\mathrm{O}-\mathrm{U}$ process.

\section{The Bivariate Trending O-U Process}

An obvious deficiency of the trending O-U process as a general model of asset prices is the fact that its returns are negatively autocorrelated at all lags, which is inconsistent with the empirical autocorrelations of many traded assets. For example, Lo and MacKinlay $(1988,1990)$ show that equity portfolios tend to be positively autocorrelated at shorter horizons, while Fama and French (1988) and Poterba and Summers (1988) find negative autocorrelation at longer horizons. Moreover, since the trending O-U's drift depends only on $q(t)$, it leaves no role for other economic variables to play in determining the predictability of asset returns. 
To address these shortcomings, we propose the "bivariate trending O-U" process in the following sections. Although it is a special case of a bivariate linear diffusion process, and is therefore extremely tractable, it exhibits a surprisingly wide variety of autocorrelation patterns (see, for example, Figure 1). Moreover, as its name suggests, the bivariate trending $\mathrm{O}-\mathrm{U}$ process allows the log-price process to depend upon a second process, which may be inter-

$$
\rho_{\tau}(1)
$$

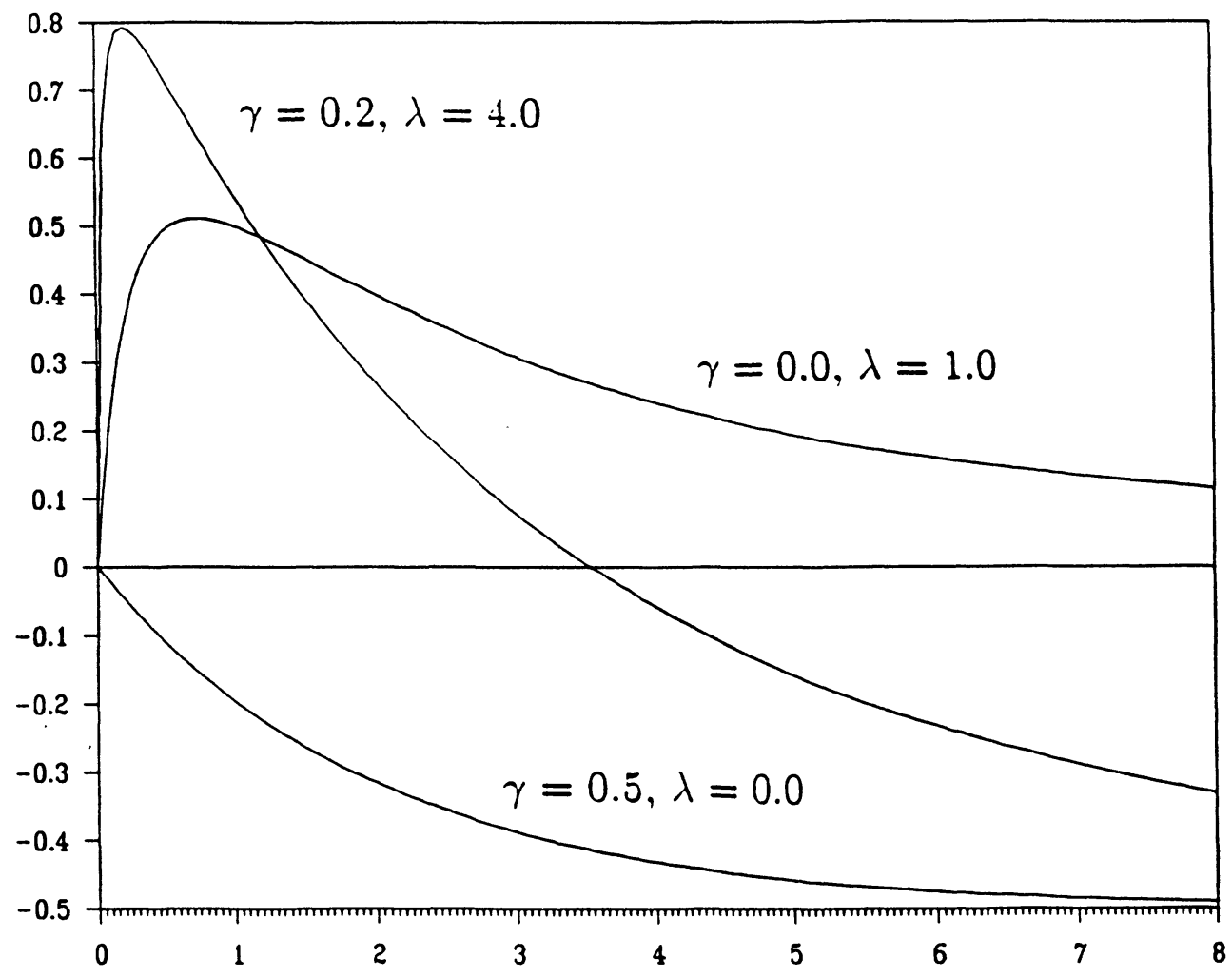

$\tau$

Figure 1. First-order autocorrelation of stock returns as a function of the length of holding period for the bivariate trending Ornstein-Uhlenbeck process. The figure plots the first-order autocorrelation of stock returns, $\rho_{\tau}(1)$, as a function of the length of holding period, $\tau$, when log-prices follow a bivariate trending Ornstein-Uhlenbeck (O-U) process. $\gamma$ is the coefficient for $q(t)$, and $\lambda$ is the coefficient for $X(t)$ in the linear drift of the detrended log-price process. $\delta$ is the mean-reversion coefficient of $X(t), \sigma$ and $\sigma_{x}$ are the diffusion coefficients for $q(t)$ and $X(t)$, respectively, and $\kappa$ is the correlation coefficient between the diffusion terms of $q(t)$ and $X(t)$. For this figure, the following parameters are fixed at: $\delta=0.6, \sigma^{2}=0.5, \sigma_{x}^{2}=0$, and $\kappa=0$. 
preted as a time-varying expected return factor that may or may not be observable.

\section{A. Basic Properties}

Let the detrended log-price process $q(t) \equiv p(t)-\mu t$ satisfy the following pair of stochastic differential equations:

$$
\begin{aligned}
& d q(t)=-(\gamma q(t)-\lambda X(t)) d t+\sigma d W_{q} \\
& d X(t)=-\delta X(t) d t+\sigma_{x} d W_{x}
\end{aligned}
$$

where $\gamma \geq 0, \delta \geq 0, q(0)=q_{0}, X(0)=X_{0}$, and $t \in[0, \infty) . W_{q}$ and $W_{x}$ are two standard Wiener processes such that $d W_{q} d W_{x}=\kappa d t$, and $X(t)$ is another stochastic process that may or may not be observable. For reasons that will become apparent below, we shall call this system the "bivariate trending O-U” process.

The bivariate system (equations (26) to (27)) contains several interesting special cases. For example, when $\lambda=0$ it reduces to the univariate trending O-U process of Section II. $A$ in which asset returns are always negatively autocorrelated. When $\gamma=0$, the drift of the detrended log-price process is $\lambda X(t)$, which is stochastic and mean reverting to its unconditional mean of zero. In the more general case when $\gamma \neq 0$, the detrended log-price process may be rewritten as:

$$
d q(t)=-\gamma\left(q(t)-\frac{\lambda}{\gamma} X(t)\right) d t+\sigma d W_{q}
$$

which shows that $q(t)$ is mean reverting to a stochastic mean $(\lambda / \gamma) X(t)$, with "speed of adjustment" $\gamma$. Since equations (26) to (27) is a system of linear stochastic differential equations, it may be solved explicitly as:

$$
\begin{aligned}
q(t)= & e^{-\gamma t} q_{0}+\frac{\lambda}{\gamma-\delta}\left[e^{-\delta t}-e^{-\gamma t}\right] X_{0} \\
& +\int_{0}^{t} e^{-\gamma(t-s)} \sigma d W_{q}(s)+\frac{\lambda}{\gamma-\delta} \int_{0}^{t}\left[e^{-\delta(t-s)}-e^{-\gamma(t-s)}\right] \sigma_{x} d W_{x}(s) \\
X(t)= & e^{-\delta t} X_{0}+\int_{0}^{t} e^{-\delta(t-s)} \sigma_{x} d W_{x}(s)
\end{aligned}
$$

where $t \geq 0$ and $q_{0}=p_{0}$ and $(q, X)$ is jointly normally distributed given its initial value $\left(q_{0}, X_{0}\right)$ at $t=0 .{ }^{16}$ From this explicit solution, the joint moments of $(q, X)$ are easily obtained and given in the Appendix.

As in the case of the univariate trending O-U process, the bivariate process is trend-stationary, the variance of its increments approaches a finite limit of $2 \operatorname{Var}[q(t)]$ and the first-order autocorrelation $\rho_{\tau}(1)$ of $\tau$-period returns ap-

\footnotetext{
${ }^{16}$ Even if $\left\{q_{0}, X_{0}\right\}$ are stochastic, as long as they are drawn from their stationary joint normal distribution, $\{q(t), X(t)\}$ is still jointly normally distributed.
} 
proaches $-\frac{1}{2}$ as $\tau$ increases without bound. Both of these restrictions are relaxed in the multivariate version of Section IV.

To see that the bivariate trending O-U process can capture more complex patterns of autocorrelation than its univariate counterpart, consider the behavior of its first-order autocorrelation function as a function of the holding period $\tau$ for the special case where $\lambda=\gamma$. As $\tau$ increases without bound, $\rho_{\tau}(1)$ approaches $-\frac{1}{2}$ as it must be for the continuously compounded $\tau$-period return of any stationary process. As $\tau$ decreases to $0, \rho_{\tau}(1)$ also approaches zero as it must for any diffusion process, since diffusions have locally independent increments by construction. For small $\tau$, we have:

$$
\rho_{\tau}(1) \approx-\frac{\tau}{2}\left(\gamma-\frac{\delta \beta_{q x}}{1-\beta_{q x}}\right),
$$

where $\beta_{q x} \equiv \operatorname{Cov}[q(t), X(t)] / \operatorname{Var}[q(t)]$. Unlike the univariate trending O-U process, in this case $\rho_{\tau}(1)$ can be either positive or negative, depending on whether $\beta_{q x}$ is greater than or less than $\gamma /(\gamma+\delta)$. Therefore, when $\beta_{q x}>\gamma /(\gamma+\delta)$, the bivariate trending O-U process will display an autocorrelation pattern that matches the empirical findings of both Lo and MacKinlay (1988) and Fama and French (1988) simultaneously: positive autocorrelation for short horizons, and negative autocorrelation for long horizons. Some other examples of first-order autocorrelation functions of the bivariate trending O-U process are given in Figure 1. For further intuition into the correlation structure of the bivariate trending O-U process, we present its general autocorrelation function in the Appendix.

\section{B. Predictability versus Autocorrelation}

We have argued in Section II that the numerical value of the Black-Scholes input $\sigma$ depends on our assumption about the data-generating process when we have discretely-sampled data. In the particular case of the univariate trending O-U process of Section II.A, the numerical value of $\sigma$ increases with the absolute value of the return autocorrelation, given a fixed numerical value for the unconditional variance of returns. However, in the case of the bivariate trending $\mathrm{O}-\mathrm{U}$ process, there is no longer such a simple relation between autocorrelation and $\sigma$.

For example, consider the special case of the bivariate trending O-U process in which $\gamma=0$, hence $\lambda X(t)$ is the drift of the detrended log-price process, and the system reduces to:

$$
\begin{aligned}
& d q(t)=\lambda X(t) d t+\sigma d W_{q} \\
& d X(t)=-\delta X(t) d t+\sigma_{x} d W_{x} .
\end{aligned}
$$

For simplicity, also let $\kappa=0$ so that $d W_{q}$ and $d W_{x}$ are statistically independent. In this special case, asset returns are positively autocorrelated at all leads and lags. We may calculate the unconditional variance and autocorrelation of returns by taking the limit of $\gamma \rightarrow 0$ in equations (A10) and (A12). 
Then, for any holding period $\tau$ we have:

$$
\begin{aligned}
s^{2}\left(r_{\tau}\right) & =\sigma^{2} \frac{1}{1-\sigma_{q x}^{*}}\left[\tau-\frac{\sigma_{q x}^{*}}{\delta}\left(1-e^{-\delta \tau}\right)\right] \\
\rho_{\tau}(1) & =\frac{\frac{\sigma_{q x}^{*}}{\delta}\left(1-e^{-\delta \tau}\right)^{2}}{2 \tau\left[1-\frac{\sigma_{q x}^{*}}{\delta \tau}\left(1-e^{-\delta \tau}\right)\right]}, \quad \text { where } \quad \sigma_{q x}^{*} \equiv \frac{1}{1+\left(\frac{\delta}{\lambda}\right)^{2} \frac{\sigma^{2}}{\sigma_{x}^{2}}} .
\end{aligned}
$$

Observe that $0<\sigma_{q x}^{*} \leq 1$, and that $\sigma_{q x}^{*}$ is an increasing function of $\lambda$. Since $\rho_{\tau}(1)$ is an increasing function of $\sigma_{q x}^{*}$, it is also an increasing function of $\lambda$. By increasing $\lambda$ while holding fixed the unconditional variance of returns, we can see the effects of increasing autocorrelation on the Black-Scholes input $\sigma$. Rearranging equation (34) yields:

$$
\sigma^{2}=\frac{1-\sigma_{q x}^{*}}{1-\frac{\sigma_{q x}^{*}}{\delta \tau}\left(1-e^{-\delta \tau}\right)} \cdot \frac{s^{2}\left(r_{\tau}\right)}{\tau}
$$

which shows that an increase in the return autocorrelation (through an increase in $\lambda$ ) is accompanied by a decrease in $\sigma$ and a corresponding decrease in the Black-Scholes call option price. ${ }^{17}$ Increasing return autocorrelation in this case has precisely the opposite effect on option prices than in the case of the univariate trending $\mathrm{O}-\mathrm{U}$ process, in which an increase in the absolute value of the return autocorrelation increases the numerical value of $\sigma$, increasing option prices (recall that in that case, the autocorrelation is always nonpositive).

While increasing autocorrelation can either increase or decrease option prices, depending on the particular specification of the drift, the special case of equations (32) and (33) does illustrate a general relation between option prices and predictability. To see this, we must first define predictability explicitly. Perhaps the most common definition is the $R^{2}$ coefficient, or the fraction of the unconditional variance of the dependent variable that is "explained" by the conditional mean or predictor. Higher $R^{2} \mathrm{~s}$ are generally taken to mean more predictability, and this interpretation is appropriate in our context with three additional restrictions:

Restriction 1: The unconditional variance of returns $r_{\tau}(t)$ is fixed.

Restriction 2: The drift is not a function of the log-price process $p(t)$.

Restriction 3: $W_{q}$ is statistically independent of $W_{x}$.

The first restriction has already been discussed above - the very nature of prediction takes as given the object to be predicted, and meaningful compar-

${ }^{17}$ It is easy to show that the expression $\left(1-\sigma_{q x}^{*}\right) /\left[1-\delta_{q x}^{*}\left(1-e^{-\delta \tau}\right) / \delta \tau\right]$ decreases as $\sigma_{q x}^{*}$ increases. It then follows that increasing $\lambda$ will increase its value since $\sigma_{q x}^{*}$ will increase. 
isons of alternate prediction equations cannot be made if the "target" is allowed to change in any way. In particular, if the unconditional variance of $r_{\tau}(t)$ is not fixed, a reduction in the prediction error variance need not imply better predictability because it may be accompanied by a more-than-proportionate reduction in the unconditional variance to be predicted.

Restrictions 2 and 3 eliminate feedback relations between the conditional mean and the prediction error or residual, so that the discrete-time representation of the continuous-time system is a genuine prediction equation, i.e., the conditional expectation of the residual, conditioned on the drift, is zero.

Under these restrictions, it may be shown that an increase in predictability - as measured by $R^{2}$-always decreases $\sigma$ and therefore decreases option prices. ${ }^{18}$ The intuition for this relation is clear: holding fixed the unconditional variance of returns, an increase in the variability of the conditional mean must imply a decrease in the variability of the residual. More formally, the unconditional variance of returns may always be written as the following sum:

$$
\operatorname{Var}\left[r_{\tau}(t)\right]=\operatorname{Var}\left[E\left[r_{\tau}(t) \mid \Omega_{t}\right]\right]+E\left[\operatorname{Var}\left[r_{\tau}(t) \mid \Omega_{t}\right]\right]
$$

where $\Omega_{t}$ is the conditioning information set. Holding the left-hand side of equation (37) fixed, an increase in the first term of the right-hand side, i.e., an increase in predictability, must be accompanied by an equal decrease in the second term of the right-hand side. Furthermore, under Restrictions 1 to 3 , the variability of the residual can be shown to be monotonically related to the continuous-time parameter $\sigma$, hence increasing predictability implies decreasing option prices.

In particular, under the bivariate trending O-U process, increasing $\lambda$ has the effect of increasing the variability of the conditional mean. Holding the unconditional variance of the returns $s^{2}\left(r_{\tau}\right)$ fixed, an increase in $\lambda$ will therefore increase the predictability of returns, implying that the value of $\sigma^{2}$ must decrease since Restrictions 1 to 3 are satisfied by equations (32) and (33). ${ }^{19}$ As $\lambda$ increases without bound so that progressively more variation in returns is attributable to the time-varying drift, returns become progressively more predictable, $\sigma$ approaches 0 and the option's value approaches its lower

${ }^{18}$ It is important to note that, here and throughout this article, we consider changes in predictability or $R^{2}$ only through changes in the specification or functional form of the drift. Another way to change predictability, which we do not consider, is to change the information set under a given specification of the continuous-time process.

${ }^{19}$ Another way to see this is to consider the special case where the conditioning information set at $t$ is the whole sample path of $X(\cdot)$ from $t$ to $t+\tau$, i.e., $\Omega_{t}=\left[X_{s}: t \leq s \leq t+\tau\right]$. In this case,

$$
\operatorname{Var}\left[\mathrm{E}\left[r_{\tau}(t) \mid \Omega_{t}\right]\right]=\left(\frac{\lambda}{\delta}\right)^{2} \sigma_{x}^{2}\left[\tau-\frac{1}{\delta}\left(1-e^{-\delta \tau}\right)\right], \quad \mathrm{E}\left[\operatorname{Var}\left[r_{\tau}(t) \mid \Omega_{t}\right]\right]=\sigma_{q}^{2} \tau .
$$

Thus, the residual variance is simply $\sigma_{q}^{2} \tau$. Increasing $\lambda$ increases the predictability in returns and decreases $\sigma^{2}$ holding $\operatorname{Var}\left[r_{\tau}(t)\right]$ fixed. We thank the referee for suggesting this special case. 
bound of $e^{-r(T-t)} \operatorname{Max}[P(T)-K, 0] .^{20}$ Only if predictability is defined in this narrow sense, and only under Restrictions 1 to 3 is there an unambiguous relation between predictability and option prices.

Under more general conditions, however, a simple relation between predictability and option prices is not available, and the very notion of predictability need not be well-defined. For example, Restriction 2 is violated by the univariate trending O-U process of Section II. $A$, and in that case, while increasing predictability does decrease the variance of the prediction error of $r_{\tau}(t)$, it also increases $\sigma$.

\section{A Numerical Example}

To illustrate the importance of predictability in determining the BlackScholes input $\sigma$, we use historical daily returns of the Center for Research in Security Prices (CRSP) value-weighted market index from 1962 to 1990 to calibrate the bivariate trending O-U process and evaluate $\sigma$ explicitly. Since all second-order moments of continuously compounded returns depend on the six underlying parameters of the bivariate process, $\gamma, \delta, \lambda, \sigma, \sigma_{x}$ and $\kappa$, we may choose any six moments and solve for the six underlying parameters. Moreover, if $\gamma \neq 0$, we can set $\lambda=\gamma$ without loss of generality, which reduces the total number of free parameters to five. To further simplify the calibration exercise, we set $\kappa=0$. Thus, we require only four second-order moments to determine $\gamma, \delta, \sigma$, and $\sigma_{x}$.

For the four second-order moments, we use the sample variance of the returns $\operatorname{Var}\left[r_{\tau}(t)\right]$, the first-order autocorrelation coefficient $\rho_{\tau}(1)$, and two higher-order autocorrelation coefficients. If the bivariate trending O-U process is the true data-generating process and we possessed the actual population values of the moments, then of course the choice of which two higher order autocorrelation coefficients to fit is arbitrary, since they will arrive at the same parameter values. However, since we are using actual data to perform the calibration and are not estimating the parameters of the system, some care is required in selecting the moments to match. In particular, since the autocorrelation function of the bivariate trending O-U process can change sign only once (from positive to negative), we must choose our moments to be consistent with this restriction. With this in mind, we select the following four moments for our calibration: $s\left(r_{\tau}\right)=0.0085, \rho_{\tau}(1)=0.1838, \rho_{\tau}(5)=$ 0.0323 , and $\rho_{\tau}(25)=-0.0092$, where $\rho_{\tau}(k)$ denotes the $k$ th order autocorrelation of $\tau$-period returns. Calibrating the parameters to these moments yield the following values: ${ }^{21} \gamma=0.3748, \delta=0.0106, \sigma_{x}=0.0128$, and $\sigma=0.0074$. Observe that the value of the Black-Scholes input $\sigma$ under the bivariate trending O-U specification, 0.0074 , is approximately 13 percent smaller than

\footnotetext{
${ }^{20}$ Note that this particular limit is economically unrealizable because even though the stock price is still stochastic when $\sigma$ vanishes (due to the drift), it is once-differentiable and therefore admits arbitrage (see Harrison, Pitbladdo, and Schaefer (1984)).

${ }^{21}$ Note that the solution for $\gamma, \delta$, and $\sigma_{x}$ is not unique; however, the solution for $\sigma$ is.
} 
the standard deviation of continuously compounded returns 0.0085 , which is the value of $\sigma$ under an arithmetic Brownian motion specification.

The theoretical call option prices for a hypothetical \$40 stock in Table IV show that such a difference can have potentially large effects, particularly for longer maturity options just as in Tables I to III. However, in this case the naive Black-Scholes prices are overestimates of the correct call price, since the $\sigma$ that accounts for predictability is lower than the $\sigma$ obtained under an assumption of independently and identically distributed (i.i.d.) returns.

\section{The Multivariate Trending O-U Process}

Despite the flexibility of the bivariate trending O-U process, as a model of asset prices it has at least three unattractive features that are both related to the behavior of its increments as the differencing interval increases without bound: the variance of its increments approaches a finite limit, its first-order autocorrelation function approaches a limit of $-\frac{1}{2}$ and can change sign only once. Moreover, the bivariate process does not allow for additional economic or "state" variables that might affect the drift.

In this section we present a multivariate extension of the bivariate trending O-U process that addresses all of these concerns. By allowing the drift to depend linearly on additional state variables, resulting in the "multivariate trending O-U process," richer patterns of autocorrelation can be captured without sacrificing tractability. If the state variables are stationary, then log-prices are trend-stationary as in the bivariate case. If the state variables are random walks, then log prices will contain stochastic trends, in which case the variance of its increments can increase without bound and the

\section{Table IV}

\section{Option Prices Under the Bivariate Trending Ornstein-Uhlenbeck Process (Daily Frequency)}

Comparison of theoretical call option prices on a hypothetical $\$ 40$ stock under an arithmetic Brownian motion versus a bivariate trending Ornstein-Uhlenbeck $(\mathrm{O}-\mathrm{U})$ process for log-prices, both calibrated to match the daily CRSP value-weighted returns index from 1962 to 1990 . The time-to-maturity is given by $T-t$, entries under the "B-S" heading are call prices calculated under the Black-Scholes assumption of arithmetic Brownian motion [for which $\sigma=0.0085$ ], and entries under the "O-U" heading are call prices calculated under the bivariate trending Ornstein-Uhlenbeck [for which $\sigma=0.0074$ ]. A daily continuously-compounded risk-free rate of $\log (1.05) / 364$ is assumed.

\begin{tabular}{|c|c|c|c|c|c|c|c|c|c|c|}
\hline \multirow{2}{*}{$\begin{array}{c}\text { Strike } \\
\text { Price }\end{array}$} & \multicolumn{2}{|c|}{$T-t=7$} & \multicolumn{2}{|c|}{$T-t=91$} & \multicolumn{2}{|c|}{$T-t=182$} & \multicolumn{2}{|c|}{$T-t=273$} & \multicolumn{2}{|c|}{$T-t=364$} \\
\hline & B-S & O-U & B-S & O-U & B-S & $\mathrm{O}-\mathrm{U}$ & B-S & $\mathrm{O}-\mathrm{U}$ & B-S & $\mathrm{O}-\mathrm{U}$ \\
\hline 30 & 10.028 & 10.028 & 10.363 & 10.363 & 10.725 & 10.722 & 11.092 & 11.080 & 11.463 & 11.438 \\
\hline 35 & 5.033 & 5.033 & 5.467 & 5.442 & 6.005 & 5.931 & 6.537 & 6.422 & 7.049 & 6.901 \\
\hline 40 & 0.378 & 0.331 & 1.542 & 1.378 & 2.328 & 2.100 & 2.990 & 2.716 & 3.586 & 3.276 \\
\hline 45 & 0.000 & 0.000 & 0.155 & 0.088 & 0.562 & 0.393 & 1.020 & 0.771 & 1.485 & 1.174 \\
\hline 50 & 0.000 & 0.000 & 0.005 & 0.001 & 0.085 & 0.038 & 0.263 & 0.148 & 0.508 & 0.320 \\
\hline
\end{tabular}


first-order autocorrelation can approach 0 as the differencing interval increases.

In a straightforward generalization of the bivariate case, we let the detrended log-price process $q(t)$ fluctuate around a stochastic mean, now governed by a multivariate linear process. Specifically, let:

$$
\begin{aligned}
d q(t) & =[-\gamma q(t)+\Lambda \mathbf{X}(t)] d t+\sigma d W_{q} \\
d \mathbf{X}(t) & =-\Delta \mathbf{X}(t) d t+\mathbf{B}_{x} d \mathbf{W}_{x}
\end{aligned}
$$

and $q(0)=q_{0}, \mathbf{X}(0)=\mathbf{X}_{0}$, and $t \in[0, \infty)$, where $\mathbf{X}(t)$ is an $m$-dimensional random process, $\mathbf{W}_{x}(t)$ is a $k$-dimensional standard Wiener process, $\gamma$ and $\sigma$ are scalar parameters, and $\Lambda, \Delta, \mathbf{B}_{x}$ are $(1 \times m),(m \times m),(m \times k)$ matrix parameters, respectively. Without loss of generality we assume that $\Delta$ is diagonal, i.e., $\Delta=\operatorname{diag}\left\{\delta_{i}\right\}$. The linear system $\left[q(t) \mathbf{X}(t)^{\prime}\right]$ defined by equations (38) and (39) has the following explicit solution:

$$
\begin{aligned}
q(t)= & e^{-\gamma t} q_{0}+\Lambda(\gamma \mathbf{I}-\Delta)^{-1}\left[e^{-\Delta t}-e^{-\gamma \mathbf{I} t}\right] \mathbf{X}_{0}+\int_{0}^{t} e^{-\gamma(t-s)} \sigma d W_{q}(s) \\
& -\Lambda(\gamma \mathbf{I}-\Delta)^{-1} \int_{0}^{t}\left[e^{-\gamma \mathbf{I}(t-s)}-e^{-\Delta(t-s)}\right] \mathbf{B}_{x} d \mathbf{W}_{x}(s) \\
\mathbf{X}(t)= & e^{-\Delta t} \mathbf{X}_{0}+\int_{0}^{t} e^{-\Delta(t-s)} \mathbf{B}_{x} d \mathbf{W}_{x}(s)
\end{aligned}
$$

where $\mathbf{I}$ is the $(m \times m)$ identity matrix. ${ }^{22}$ Since $\Delta$ is diagonal, $e^{-\Delta(t-s)}=$ $\operatorname{diag}\left\{e^{-\delta_{t}(t-s)}\right\}$. Given equations (40) and (41), we can readily derive the unconditional moments of $q(t)$ and $\mathbf{X}(t)$ (if they exist), as well as those of returns over any finite holding period $\tau^{23}$.

If the diagonal matrix $\Delta$ contains strictly positive diagonal entries $\delta_{i}$, then the log-price process is trend-stationary as in the case of the bivariate trending O-U process. The unconditional moments of the detrended log-price process and returns follow analogously from equations (40) and (41) and some of these are reported in the Appendix.

Alternatively, if at least one of the state variables follows a random walk or is "difference-stationary" (and there are no degeneracies due to cointegration), then the log-price process will also be difference-stationary and the variance of its increments will increase without bound as the differencing interval approaches infinity. For example, consider the following trivariate special case of equation (39). Let $\mathbf{X}(t) \equiv[X(t) Z(t)]^{\prime}, \Delta=\operatorname{diag}\left(\delta, \delta_{z}\right), \delta_{z}=0, \mathbf{B}_{x}=$ $\operatorname{diag}\left(\sigma_{x}, \sigma_{z}\right)$, and $d \mathbf{W}_{x}=\left[d W_{x} d W_{z}\right]^{\prime}$. In this case, $X(t)$ follows an O-U process while $Z(t)$ follows a random walk. Assume that $\gamma>0$. Without loss of

\footnotetext{
${ }^{22}$ We have implicitly assumed that $\gamma \neq \delta_{i}, i=1, \ldots, m$ so that the inverse of $\gamma \mathbf{I}-\Delta$ exists. If not, we can derive the corresponding solution by taking the appropriate limit.

${ }^{23}$ As in the bivariate case, when $\Delta$ is not of full rank, i.e., when $\delta_{i}=0$ for some $i$, or when $\gamma=0$, the unconditional moments of $q(t)$ no longer exist. However, the unconditional moments of returns do exist, and they can be calculated by taking the appropriate limits; see the discussion in Section IV.
} 
generality, we can let $\Lambda=\left[\begin{array}{ll}\gamma & \gamma\end{array}\right]$. Then the explicit solution for the detrended price process $q(t)$ is:

$$
\begin{aligned}
q(t)= & \tilde{q}(t)+Z(t) \\
\tilde{q}(t)= & e^{-\gamma t} \tilde{q}_{0}+\frac{\gamma}{\gamma-\delta}\left(e^{-\delta t}-e^{-\gamma t}\right) X_{0}+\int_{0}^{t} e^{-\gamma(t-s)} \sigma d W_{q}(s) \\
& \quad-\int_{0}^{t} e^{-\gamma(t-s)} \sigma_{z} d W_{z}(s)+\frac{\gamma}{\gamma-\delta} \int_{0}^{t}\left[e^{-\delta(t-s)}-e^{-\gamma(t-s)}\right] \sigma_{x} d W_{x}(s) \\
X(t)= & e^{-\delta t} X_{0}+\int_{0}^{t} e^{-\delta(t-s)} \sigma_{x} d W_{x}(s) \\
Z(t)= & Z_{0}+\int_{0}^{t} \sigma_{z} d W_{z}(s)
\end{aligned}
$$

Observe that $q(t)$ can be decomposed into two components: a stationary component $\tilde{q}(t)$ and a random walk component $Z(t)$, where the stationary component $\tilde{q}(t)$ behaves like the detrended log-price in the stationary bivariate $\mathrm{O}-\mathrm{U}$ case. However, in contrast to the trend-stationary case, the existence of a random walk component in the detrended log-price implies that the risk of holding the asset increases with the holding period.

Of course, when $q(t)$ is nonstationary, the unconditional moments of $q(t)$ are no longer well defined. However, the unconditional moments of the increments of $q(t)$, which are simply the de-meaned continuously compounded returns, are well defined and may be obtained from the results for the stationary case by taking the limit $\delta_{z} \rightarrow 0$.

\section{Maximum Likelihood Estimation}

The fact that the univariate and the bivariate trending O-U processes imply such different relations between autocorrelation and option values illustrates the complexity and importance of correctly identifying the datagenerating process before implementing an option pricing formula. In the previous sections, we have shown that, holding fixed the unconditional moments of the true data-generating process, a change in the specification of the drift can change the population value of the Black-Scholes input $\sigma$. As a result, a change in the specification of the drift can also change the empirical estimate of $\sigma$.

Perhaps the most direct approach to addressing these issues is to propose a reasonably flexible specification of the drift that can capture a wide variety of autocorrelation patterns, derive the exact discrete-time representation of the log-price process, estimate all the parameters of this discrete-time process simultaneously, and then solve for the parameters of the continuous-time process - which includes $\sigma$-as a function of the parameter estimates of the discretely sampled data. Since all three of our specifications for the drift are linear, their discrete-time representations are readily available and are also 
linear processes, to which maximum likelihood estimation may be applied, as described in Lo $(1986,1988)$.

To this end, denote by $t_{k}$ the sampling dates, where $k=1,2, \ldots, n$, and let $t_{k}-t_{k-1}=\tau$ be a constant, hence $t_{k}=k \tau .^{24}$ Let $q_{k} \equiv q\left(t_{k}\right)=p\left(t_{k}\right)-\mu t_{k}$ and assume that $q_{k}$ is observed. Of course, in practice the trend rate $\mu$ must be estimated, but as long as a consistent estimator of $\mu$ is available, replacing $\mu$ with $\hat{\mu}$ will have no effect upon the asymptotic properties of the parameter estimates.

\section{A. Estimating the Univariate Trending $O$-U Process}

From the explicit solution of equation (9) of the univariate trending O-U process, it is easy to obtain a recursive representation of $q_{k}$ which shows that its deviations from trend follow an $\mathrm{AR}(1)$ :

$$
q_{k}=e^{-\gamma \tau} q_{k-1}+\epsilon_{k}, \quad \epsilon_{k} \equiv \sigma \int_{t_{k-1}}^{t_{k}} e^{-\gamma\left(t_{k}-s\right)} d W(s) .
$$

For this simple process, the maximum likelihood estimator of the discrete-time parameters is asymptotically equivalent to the ordinary least squares estimator applied to detrended log-prices. The continuous-time parameters $\mu, \sigma$, and $\gamma$ may then be obtained from the discrete-time parameter estimates.

\section{B. Estimating the Bivariate Trending $O-U$ Process}

Let $X_{k} \equiv X\left(t_{k}\right)$. Then from equations (26) and (27), we have:

$$
\begin{aligned}
& q_{k}=\alpha_{q} q_{k-1}+\phi X_{k-1}+\epsilon_{q, k} \\
& X_{k}=\alpha_{x} X_{k-1}+\epsilon_{x, k},
\end{aligned}
$$

where $\alpha_{q} \equiv e^{-\gamma \tau}, \alpha_{x} \equiv e^{-\delta \tau}, \phi \equiv(\lambda /(\gamma-\delta))\left(\alpha_{x}-\alpha_{q}\right)$, and

$$
\begin{aligned}
\epsilon_{q, k} & \equiv \int_{t_{k-1}}^{t_{k}} e^{-\gamma\left(t_{k}-s\right)} \sigma d W_{q}(s)+\frac{\lambda}{\gamma-\delta} \int_{t_{k-1}}^{t_{k}}\left[e^{-\delta\left(t_{k}-s\right)}-e^{-\gamma\left(t_{k}-s\right)}\right] \sigma_{x} d W_{x}(s) \\
\epsilon_{x, k} & \equiv \int_{t_{k-1}}^{t_{k}} e^{-\delta\left(t_{k}-s\right)} \sigma_{x} d W_{x}(s) .
\end{aligned}
$$

Observe that $\left[\begin{array}{lll}\epsilon_{q, k} & \epsilon_{x, k}\end{array}\right]^{\prime}$ is a bivariate normal vector that is temporally independently and identically distributed, with mean 0 and covariance matrix $\mathbf{S}_{\tau}$ given in the Appendix. Rewriting equations (47) and (48) in vector form yields:

$$
\left(\begin{array}{c}
q_{k} \\
X_{k}
\end{array}\right)=\left(\begin{array}{cc}
\alpha_{q} & \phi \\
0 & \alpha_{x}
\end{array}\right)\left(\begin{array}{c}
q_{k-1} \\
X_{k-1}
\end{array}\right)+\left(\begin{array}{c}
\epsilon_{q, k} \\
\epsilon_{x, k}
\end{array}\right) .
$$

${ }^{24}$ This last assumption is made purely for notational convenience-irregularly sampled data may be just as easily accommodated but is notationally more cumbersome. 
This is simply a bivariate AR(1) process, where the second component, $X_{k}$, may or may not be observed. The parameters of this discrete-time process may be estimated by maximum likelihood by casting equation (51) in statespace form and applying the Kalman filter (e.g., Harvey (1989a) or Lütkepohl (1991)). There are seven parameters to be estimated: $\mu, \alpha_{q}, \alpha_{x}, \phi$, and the elements of the symmetric $(2 \times 2)$ matrix $\mathbf{S}_{\tau}$. From the definition of these discrete-time parameters, we can uniquely determine the seven parameters of the underlying continuous-time process, $\mu, \gamma, \delta, \lambda, \sigma, \sigma_{x}$, and $\kappa$ (see equations (A21), (A22), (A23), and (A24) in the Appendix), hence the principle of invariance yields maximum likelihood estimators for these as well.

\section{Estimating the Multivariate Trending $O-U$ Process}

The discrete-time representation of equations (38) and (39) is a straightforward generalization of the bivariate case:

$$
\begin{aligned}
q_{k} & =\alpha_{q} q_{k-1}+\Phi \mathbf{X}_{k-1}+\epsilon_{q, k} \\
\mathbf{X}_{k} & =\mathbf{A}_{x} \mathbf{X}_{k-1}+\epsilon_{x, k}
\end{aligned}
$$

where $q_{k} \equiv q\left(t_{k}\right), \mathbf{X}_{k} \equiv \mathbf{X}\left(t_{k}\right), \alpha_{q} \equiv e^{-\gamma \tau}, \mathbf{A}_{x} \equiv e^{-\Delta \tau}, \Phi=\Lambda(\gamma \mathbf{I}-\Delta)^{-1}\left(\mathbf{A}_{x}-\right.$ $\left.\alpha_{q} \mathbf{I}\right)$, and

$$
\begin{aligned}
\epsilon_{q, k} \equiv & \int_{t_{k-1}}^{t_{k}} e^{-\gamma\left(t_{k}-s\right)} \sigma d W_{q}(s) \\
& -\Lambda(\gamma \mathbf{I}-\Delta)^{-1} \int_{t_{k-1}}^{t_{k}}\left[e^{-\gamma \mathbf{I}\left(t_{k}-s\right)}-e^{-\Delta\left(t_{k}-s\right)}\right] \mathbf{B}_{x} d \mathbf{W}_{x}(s) \\
\epsilon_{x, k} \equiv & \int_{t_{k-1}}^{t_{k}} e^{-\Delta\left(t_{k}-s\right)} \mathbf{B}_{x} d \mathbf{W}_{x}(s) .
\end{aligned}
$$

Observe that $\mathbf{e}_{k} \equiv\left[\begin{array}{ll}\epsilon_{q, k} & \epsilon_{x, k}^{\prime}\end{array}\right]^{\prime}$ is an $(m+1)$-dimensional normal random variable which is temporally independently and identically distributed. In vector form, we have:

$$
\left(\begin{array}{c}
q_{k} \\
\mathbf{X}_{k}
\end{array}\right)=\left(\begin{array}{cc}
\alpha_{q} & \Phi \\
0 & \mathbf{A}_{x}
\end{array}\right)\left(\begin{array}{c}
q_{k-1} \\
\mathbf{X}_{k-1}
\end{array}\right)+\mathbf{e}_{k} .
$$

which is a $\operatorname{VAR}(1)$, and given observations $\left\{p_{k}\right\}$, or $\left\{p_{k}\right\}$ and some components of $\left\{\mathbf{X}_{k}\right\}$, we can obtain maximum likelihood estimates of its parameters by applying the Kalman filter to the state-space representation as before. ${ }^{25}$

In our trivariate example, equation (42) of Section IV, which is difference stationary, the discrete-time representation of equations (42) to (45) is:

$$
\begin{aligned}
\left(q_{k}-Z_{k}\right) & =\alpha_{p}\left(q_{k-1}-Z_{k-1}\right)+\phi X_{k-1}+\epsilon_{\tilde{q}, k} \\
X_{k} & =\alpha_{x} X_{k-1}+\epsilon_{x, k} \\
Z_{k} & =Z_{k-1}+\epsilon_{z, k},
\end{aligned}
$$

${ }^{25}$ However, in the general multivariate case, identification is not guaranteed and is often difficult to verify. See Lütkepohl (1991, Chapter 13.4.2) for further discussion. 
where $Z_{k} \equiv Z\left(t_{k}\right)$, and $\epsilon_{\tilde{q}, k}, \epsilon_{x, k}$, and $\epsilon_{z, k}$ are i.i.d. Gaussian shocks derived from the stochastic intervals in equations (43), (44), and (45). Since $q_{k}$ is nonstationary here, prices cannot be used directly to estimate the parameters. Instead, de-meaned continuously compounded returns may be used since they are stationary under this current specification. Define $\tilde{r}_{k} \equiv q_{k}-$ $q_{k-1}, v_{k} \equiv X_{k}-X_{k-1}$, and $\mathbf{e}_{k} \equiv\left[\begin{array}{lll}\epsilon_{\tilde{q}, k} & \epsilon_{x, k} & \epsilon_{z, k}\end{array}\right]^{\prime}$. We then have:

$$
\left(\begin{array}{c}
\tilde{r}_{k} \\
v_{k}
\end{array}\right)=\left(\begin{array}{cc}
\alpha_{p} & \phi \\
0 & \alpha_{x}
\end{array}\right)\left(\begin{array}{c}
\tilde{r}_{k-1} \\
v_{k-1}
\end{array}\right)+\left(\begin{array}{ccc}
1 & 0 & 1 \\
0 & 1 & 0
\end{array}\right) \mathbf{e}_{k}-\left(\begin{array}{ccc}
1 & 0 & -\alpha_{p} \\
0 & 1 & 0
\end{array}\right) \mathbf{e}_{k-1}
$$

which is simply a multivariate $\operatorname{ARMA}(1,1)$ process. Once again, given observations $\left\{r_{k}\right\}$, or $\left\{r_{k}, v_{k}\right\}$ maximum likelihood estimation of the discrete-time parameters may be readily performed as in the trend-stationary case via its state-space representation.

\section{Extensions and Other Issues}

There are several other aspects of the impact of predictability on option prices that deserve further discussion, such as extensions to option pricing models other than the Black-Scholes model, implications of the distinction between discrete and continuous time, the relation of our findings to those surrounding "estimation risk," and the interpretation of implied volatilities in the presence of predictability. We shall consider each of these issues in turn in the following sections.

\section{A. Extensions to Other Derivative Pricing Models}

Although we have confined our attention so far to the case where the diffusion coefficient $\sigma$ is constant-the Black-Scholes case-predictability can affect other option and derivative pricing formulas in a similar fashion. Since analytical derivative pricing formulas are almost always obtained from no-arbitrage conditions, the drift plays no role in determining the formula but plays a critical role in determining both the population values and empirical estimates of the parameters that enter the formula as arguments. For example, although the drift does not enter into Merton's (1976a) jump-diffusion option pricing formula, its specification will affect the values of $\sigma$ (the volatility of the diffusion component), $\delta$ (the volatility of the logarithm of the jump magnitude), $k$ (the expectation of the logarithm of the jump magnitude), and $\lambda$ (the mean rate of occurrence of the Poisson jump). Since all of our drift specifications in Sections II, III, and IV are linear, they may be readily incorporated into more complex stochastic processes.

\section{B. Discrete versus Continuous Time}

Clearly, the importance of the drift in implementing option pricing formulas comes from the fact that the data are sampled at discrete time intervals, while the theoretical models are formulated in continuous time. Now it is well 
known that the diffusion coefficient is a "sample-path property," so that any single realization of a continuous sample path over a finite interval is sufficient to reveal the true value of $\sigma$. However, continuous sample paths are practically unrealizable; therefore, we are always confronted with some sampling error in our attempts to estimate $\sigma$. Assuming for the moment that the diffusion coefficient $\sigma$ is indeed constant, the sampling error of any estimator of $\sigma$ can be traced to two distinct sources: misspecification of the drift and the discreteness of the sampling interval.

Of course, these two sources of sampling error are closely related. For example, the effects of misspecifying the drift diminishes as the sampling frequency increases, and in the limit there is no sampling error in estimating $\sigma$, hence misspecification of the drift is irrelevant. In particular, consider the relation between the continuous-time parameter $\sigma^{2}$ and the finite holdingperiod return variance $s^{2}\left(r_{\tau}\right)$ for the univariate trending O-U case of Section II. $A$. For any fixed value of $\sigma^{2}$, equation (18) shows that as the return horizon $\tau$ decreases to 0 , the ratio of $\sigma^{2}$ to $s^{2}\left(r_{\tau}\right) / \tau$ approaches 1 , hence $\sigma^{2}$ may be recovered exactly in the limit of continuous sampling. This is a general property of diffusions (equation (1)) with a constant diffusion coefficient - the unconditional variance $s^{2}\left(r_{\tau}\right)$ approaches $[d q(t)]^{2}=\sigma^{2} d t$ as the holding period $\tau$ approaches zero. ${ }^{26}$ Alternatively, $\sigma^{2} d t$ may be viewed as the conditional variance of $d q$, conditional on the drift. But since all the infinitesimal variation in $d q$ is attributable to the diffusion term $\sigma^{2} d W$ (recall that the drift is of order $d t$ and the diffusion term is of order $\sqrt{d t}$ ), the conditional and unconditional variance of the stochastic differential $d q$ are effectively the same (see Sims (1984) for further details).

This limiting result may lead some to advocate using the most finely sampled data available to compute $s^{2}\left(r_{\tau}\right) / \tau$, so as to minimize the effects of the drift of the data-generating process. Of course, whether or not the most finely sampled data available is fine enough to render $s^{2}\left(r_{\tau}\right) / \tau$ an adequate approximation to $\sigma^{2}$ is an empirical issue that depends critically on what the true data-generating process is and on the types of market microstructure effects that may come into play.

It is also conceivable that the sampling error in $\hat{\sigma}$ induced by a misspecification of the drift is not nearly so great as the sampling error induced by discrete sampling. While specifying a "better" drift may yield a closer approximation to the continuous-time process, it may not improve the performance of $\hat{\sigma}$ for a given set of discretely sampled data.

The potential importance of both sources of sampling error are, of course, empirical issues that must be resolved on an individual basis with a particular application and dataset at hand. For example, consider the univariate trending O-U process of Section II.A, and recall from equations (20) to (22) that $\Delta\left(1, \tau, \rho_{\tau}(1)\right)$ provides a convenient measure of the impact of serial

\footnotetext{
${ }^{26}$ In fact, even if the diffusion coefficient is time varying, it may be estimated with arbitrary precision by sampling more frequently within a fixed time span. See Huang and Lo (1994) for further details.
} 
correlation on the Black-Scholes input $\sigma^{2}$ as a function of the first-order autocorrelation coefficient $\rho_{\tau}(1)$ for $\tau$-period returns.

For example, let $s^{2}\left(r_{1}\right)$ be defined for daily returns, and suppose that the first-order autocorrelation of daily returns is -30 percent. Table $\mathrm{V}$ shows that $\Delta(1,1,-0.30)=1.527$, hence the value of $s^{2}\left(r_{1}\right)$ must be increased by 52.7 percent to yield the correct value for the Black-Scholes input $\sigma^{2}{ }^{27}$ If, however, a -30 percent first-order autocorrelation is observed for 5-day returns, this should yield a smaller autocorrelation for daily returns (recall that in the limit, the autocorrelation vanishes), which is confirmed by Table V's entry of 1.094 for $\Delta(1,5,-0.30)$, i.e., $\sigma^{2}$ is only 9.4 percent larger than $s^{2}\left(r_{1}\right)$ in this case. Even in the extreme case of a -45 percent autocorrelation, if this autocorrelation is for 25-day returns, $\sigma^{2}$ is only 4.7 percent larger than $s^{2}\left(r_{1}\right)$, whereas the same autocorrelation for daily returns implies that $\sigma^{2}$ is 156 percent larger than $s^{2}\left(r_{1}\right)$. Contrary to conventional wisdom, the autocorrelation coefficient is not unitless, and has an often-neglected time dimension to it.

A second method of gauging the relative importance of a misspecification of the drift in the sampling error of $\hat{\sigma}$ in the case of the univariate trending $\mathrm{O}-\mathrm{U}$ process is to perform a simple Monte Carlo simulation experiment. For a given sample size, say 250 observations, consider simulating a sample path of daily returns under the univariate trending O-U specification, estimating $\sigma$ with and without an adjustment for the drift, and repeating this 5,000 times to obtain the finite-sample distribution of the two estimators.

Table VI reports the outcome of such an experiment for sample sizes ranging from 250 to 1,250 daily observations (roughly 1 to 5 years of daily data), and for first-order autocorrelations $\rho_{1}$ ranging from -10 to -45 percent, and holding the daily unconditional variance fixed at 2 percent. The first two columns report the parameter values of the simulation-note that $\sigma$ changes with $\rho_{1}$ since we have fixed the daily unconditional variance at 2 percent. Columns 3 and 4 report the percentage bias of the naive estimator $\hat{s}$ (which is simply the sample standard deviation of daily returns) and the estimator $\hat{\sigma}$ that adjusts for the mean-reverting drift (based on equation (19)), where the expected value of each of the two estimators is computed over the 5,000 replications of each simulation.

The first row of Panel A of Table VI shows that $\hat{s}$ is considerably more biased than $\hat{\sigma}:-5.1$ percent for $\hat{s}$ versus 0.5 percent for $\hat{\sigma}$. For more extreme values of $\rho_{1}$, the biases of both estimators worsen, but even in the worst case when $\rho_{1}$ is -45 percent, $\hat{s}$ is more biased than $\hat{\sigma}:-37.3$ versus 12.1 percent. The relative performance of $\hat{s}$ and $\hat{\sigma}$ is similar for larger sample sizes.

Table VI also reports the theoretical value of $\Delta$, which relates $\sigma$ to $s$ according to equation (20), and its expectation over each of the 5,000 replica-

\footnotetext{
${ }^{27}$ An alternate interpretation of the entries in Table $\mathrm{V}$ is the bias in using implied volatility as an estimator of the standard deviation of returns when the trending O-U process is the data-generating process. For example, the entry 1.527 that corresponds to $\Delta(1,1,-0.30)$ suggests that when asset returns have a serial correlation coefficient of -30 percent, the upward bias in implied volatility as an estimator of the standard deviation is 52.7 percent.
} 
Table V

\section{Ratio of Instantaneous Variance $\sigma^{2}$ to the Variance of Returns $s^{2}\left(r_{1}\right)$ under the Trending \\ Ornstein-Uhlenbeck Process}

Ratio of $\sigma^{2}$ to the variance of returns $s^{2}\left(r_{1}\right)$ under the trending Ornstein-Uhlenbeck (O-U) process, for various values of the first-order autocorrelation $\rho_{\tau}(1)$ and holding period $\tau$, where $\tau$ is measured in units of the holding period used to construct $s^{2}\left(r_{1}\right)$.

\begin{tabular}{|c|c|c|c|c|c|c|c|}
\hline \multirow[b]{2}{*}{$\tau$} & \multicolumn{7}{|c|}{$\rho_{\tau}(1)$} \\
\hline & 0.00 & -0.05 & -0.10 & -0.20 & -0.30 & -0.40 & -0.45 \\
\hline 1 & 1.000 & 1.054 & 1.116 & 1.277 & 1.527 & 2.012 & 2.558 \\
\hline 2 & 1.000 & 1.027 & 1.057 & 1.133 & 1.247 & 1.456 & 1.684 \\
\hline 3 & 1.000 & 1.018 & 1.038 & 1.088 & 1.160 & 1.292 & 1.432 \\
\hline 4 & 1.000 & 1.013 & 1.028 & 1.065 & 1.119 & 1.215 & 1.315 \\
\hline 5 & 1.000 & 1.011 & 1.022 & 1.052 & 1.094 & 1.170 & 1.248 \\
\hline 6 & 1.000 & 1.009 & 1.019 & 1.043 & 1.078 & 1.140 & 1.204 \\
\hline 7 & 1.000 & 1.008 & 1.016 & 1.037 & 1.067 & 1.119 & 1.173 \\
\hline 8 & 1.000 & 1.007 & 1.014 & 1.032 & 1.058 & 1.104 & 1.151 \\
\hline 9 & 1.000 & 1.006 & 1.012 & 1.029 & 1.052 & 1.092 & 1.133 \\
\hline 10 & 1.000 & 1.005 & 1.011 & 1.026 & 1.047 & 1.083 & 1.120 \\
\hline 11 & 1.000 & 1.005 & 1.010 & 1.023 & 1.042 & 1.075 & 1.108 \\
\hline 12 & 1.000 & 1.004 & 1.009 & 1.021 & 1.039 & 1.069 & 1.099 \\
\hline 13 & 1.000 & 1.004 & 1.009 & 1.020 & 1.036 & 1.063 & 1.091 \\
\hline 14 & 1.000 & 1.004 & 1.008 & 1.018 & 1.033 & 1.059 & 1.084 \\
\hline 15 & 1.000 & 1.004 & 1.007 & 1.017 & 1.031 & 1.055 & 1.079 \\
\hline 16 & 1.000 & 1.003 & 1.007 & 1.016 & 1.029 & 1.051 & 1.074 \\
\hline 17 & 1.000 & 1.003 & 1.007 & 1.015 & 1.027 & 1.048 & 1.069 \\
\hline 18 & 1.000 & 1.003 & 1.006 & 1.014 & 1.026 & 1.045 & 1.065 \\
\hline 19 & 1.000 & 1.003 & 1.006 & 1.014 & 1.024 & 1.043 & 1.062 \\
\hline 20 & 1.000 & 1.003 & 1.006 & 1.013 & 1.023 & 1.041 & 1.059 \\
\hline 21 & 1.000 & 1.003 & 1.005 & 1.012 & 1.022 & 1.039 & 1.056 \\
\hline 22 & 1.000 & 1.002 & 1.005 & 1.012 & 1.021 & 1.037 & 1.053 \\
\hline 23 & 1.000 & 1.002 & 1.005 & 1.011 & 1.020 & 1.035 & 1.051 \\
\hline 24 & 1.000 & 1.002 & 1.005 & 1.011 & 1.019 & 1.034 & 1.049 \\
\hline 25 & 1.000 & 1.002 & 1.004 & 1.010 & 1.018 & 1.033 & 1.047 \\
\hline
\end{tabular}

tions. For most sample sizes and values of $\rho_{1}, \Delta$ and $\hat{\Delta}$ are fairly close, which explains the superiority of $\hat{\sigma}$ to $\hat{s}$.

A somewhat more subtle issue surrounding the distinction between discrete and continuous time is the fact that while we have used the BlackScholes formula to gauge the effects of asset return predictability on option prices, it may be argued that the Black-Scholes formula holds only if continuous trading is possible and costless. Indeed, to implement the replicating strategy literally requires observing the sample path of prices continuously, which eliminates the need for estimating $\sigma$ altogether. In this case, the relation between predictability and option prices still exists but is irrelevant since the true $\sigma$ can always be recovered exactly.

However, the continuous trading assumption underlying the pricing formulas does not invalidate our main conclusion: whenever option pricing formulas are implemented with discretely sampled data, the drift matters. 
Of course, ideally we should also incorporate the effect of discreteness into the pricing formulas to provide a complete and empirically relevant theory of option pricing. One approach is to simply impose discrete trading, e.g., Black and Scholes (1972) and Boyle and Emanuel (1980). Another approach is to take into account directly the economic causes of discrete trading, such as transactions costs, in constructing replicating strategies, e.g., Leland (1985). These approaches will yield either approximate pricing formulas or bounds for option prices, and, in both cases, the results will certainly depend on the numerical value of the diffusion coefficient $\sigma$ which in turn will depend on the specification of the drift, ceteris paribus. Therefore, despite the fact that in the continuous-time limit $\sigma$ becomes known, any empirical implementation must incorporate the effects of predictability on option prices.

\section{Estimation Risk}

The effects of predictability on option prices are closely related to, but not synonymous with, the problem of "estimation risk" (e.g., Barry, French, and Rao (1991)). As we discuss in Section VI. $B$, the fact that $\sigma^{2}$ must be estimated from discretely sampled data provides the primary motivation for our analysis. But the link between $\sigma^{2}$ and asset return predictability exists even when $\sigma^{2}$ is known without error. Of course, if $\sigma^{2}$ is known, then the degree of predictability in asset returns is irrelevant for purposes of pricing options even if the link is present. However, when $\sigma^{2}$ is unknown, the precise form of asset return predictability will affect both the estimate and the estimation risk of $\sigma^{2}$.

\section{Implied Volatilities}

A consequence of the Black-Scholes model is that the parameter $\sigma$ may be recovered from option prices directly by inverting equation (3). Therefore, why go through the trouble of relating asset return predictability to $\sigma$ ? The response to this simple but perplexing question is quite straightforward. The relevance of the implied volatility relies on the proper specification of the option pricing formula. If prices were truly Black-Scholes prices, then implied volatilities would be irrelevant, since the Black-Scholes model requires that $\sigma$ is known. But if the market price is not truly a Black-Scholes price, then an implied volatility obtained from the Black-Scholes formula is difficult to interpret and use.

Alternatively, for the Black-Scholes formula to yield the correct prices, some investors must know the true value of $\sigma$ (or equivalently, must observe a continuous sample path of the stock price over a finite time interval) and must be able to trade continuously. If these conditions held, other investors could obtain the true value of $\sigma$ by simply inverting the Black-Scholes formula. If, however, all investors do not know the true value of $\sigma$ but only observe (and trade at) prices at discrete time intervals, then they will have to infer the value of $\sigma$ from the data instead of the Black-Scholes formula, which no longer holds exactly. This is the situation we consider here. In this case, the market prices of options do not provide any information about the 
Table VI

\section{Impact of Estimation Error on the Autocorrelation Adjustment For Volatility o Under the Trending Ornstein-Uhlenbeck Process}

Monte Carlo simulation of the sample standard deviation estimator $\hat{s}$ and autocorrelationadjusted estimator $\hat{\sigma}$ of Black-Scholes volatility input $\sigma$ for various sample sizes and first-order autocorrelation coefficients $\rho_{1}$. Each row corresponds to an independent simulation of 5,000 sample paths, where each path is generated according to a univariate trending Ornstein-Uhlenbeck $(\mathrm{O}-\mathrm{U})$ process calibrated to daily returns with a standard deviation of 2 percent per day. Note that $\sigma$ changes with $\rho_{1}$ because the unconditional standard deviation is fixed at 2 percent per day for all the simulations. Standard errors (SE) are given in parentheses.

\begin{tabular}{|c|c|c|c|c|c|}
\hline$\rho_{1}$ & $\sigma \times 100$ & $\begin{array}{l}\text { Percentage } \\
\text { Bias }(\hat{s}) \\
(\mathrm{SE})\end{array}$ & $\begin{array}{l}\text { Percentage } \\
\text { Bias }(\hat{\sigma}) \\
(\mathrm{SE})\end{array}$ & $\sqrt{\Delta}$ & $\begin{array}{c}\sqrt{E[\hat{\Delta}]} \\
(\mathrm{SE})\end{array}$ \\
\hline \multicolumn{6}{|c|}{ Panel A. Sample Size $=250$} \\
\hline-0.10 & 2.1126 & $\begin{array}{c}-5.1488 \\
(0.0614)\end{array}$ & $\begin{array}{c}0.5048 \\
(0.0852)\end{array}$ & 1.0563 & $\begin{array}{c}1.0377 \\
(0.0597)\end{array}$ \\
\hline-0.20 & 2.2601 & $\begin{array}{r}-11.4339 \\
(0.0598)\end{array}$ & $\begin{array}{c}0.4501 \\
(0.1000)\end{array}$ & 1.1301 & $\begin{array}{c}1.1349 \\
(0.0406)\end{array}$ \\
\hline-0.30 & 2.4716 & $\begin{array}{r}-19.0601 \\
(0.0569)\end{array}$ & $\begin{array}{c}0.7178 \\
(0.1263)\end{array}$ & 1.2358 & $\begin{array}{c}1.2458 \\
(0.0528)\end{array}$ \\
\hline-0.40 & 2.8368 & $\begin{array}{r}-29.4781 \\
(0.0515)\end{array}$ & $\begin{array}{c}3.6719 \\
(0.2851)\end{array}$ & 1.4184 & $\begin{array}{c}1.4378 \\
(0.0908)\end{array}$ \\
\hline-0.45 & 3.1990 & $\begin{array}{r}-37.3341 \\
(0.0469)\end{array}$ & $\begin{array}{l}12.1053 \\
(0.04813)\end{array}$ & 1.5995 & $\begin{array}{c}1.5053 \\
(0.1279)\end{array}$ \\
\hline \multicolumn{6}{|c|}{ Panel B. Sample Size $=500$} \\
\hline-0.10 & 2.1126 & $\begin{array}{c}-5.3581 \\
(0.0439)\end{array}$ & $\begin{array}{c}0.0625 \\
(0.0610)\end{array}$ & 1.0563 & $\begin{array}{c}1.0531 \\
(0.0415)\end{array}$ \\
\hline-0.20 & 2.2601 & $\begin{array}{r}-11.3826 \\
(0.0414)\end{array}$ & $\begin{array}{c}0.3298 \\
(0.0679)\end{array}$ & 1.1301 & $\begin{array}{c}1.1326 \\
(0.0336)\end{array}$ \\
\hline-0.30 & 2.4716 & $\begin{array}{r}-19.1139 \\
(0.0395)\end{array}$ & $\begin{array}{c}0.2136 \\
(0.0844)\end{array}$ & 1.2358 & $\begin{array}{c}1.2396 \\
(0.0427)\end{array}$ \\
\hline-0.40 & 2.8368 & $\begin{array}{r}-29.5296 \\
(0.0364)\end{array}$ & $\begin{array}{c}1.1622 \\
(0.1465)\end{array}$ & 1.4184 & $\begin{array}{c}1.4348 \\
(0.0689)\end{array}$ \\
\hline-0.45 & 3.1990 & $\begin{array}{r}-37.4316 \\
(0.0333)\end{array}$ & $\begin{array}{c}6.4146 \\
(0.3412) \\
\end{array}$ & 1.5995 & $\begin{array}{c}1.5886 \\
(0.1148) \\
\end{array}$ \\
\hline \multicolumn{6}{|c|}{ Panel C. Sample Size $=750$} \\
\hline-0.10 & 2.1126 & $\begin{array}{c}-5.3334 \\
(0.0354)\end{array}$ & $\begin{array}{c}0.0766 \\
(0.0492)\end{array}$ & 1.0563 & $\begin{array}{c}1.0566 \\
(0.0303)\end{array}$ \\
\hline-0.20 & 2.2601 & $\begin{array}{r}-11.4443 \\
\quad(0.0333)\end{array}$ & $\begin{array}{c}0.1975 \\
(0.0560)\end{array}$ & 1.1301 & $\begin{array}{c}1.1317 \\
(0.0301)\end{array}$ \\
\hline-0.30 & 2.4716 & $\begin{array}{r}-19.0865 \\
(0.0326)\end{array}$ & $\begin{array}{c}0.1785 \\
(0.0683)\end{array}$ & 1.2358 & $\begin{array}{c}1.2385 \\
(0.0380)\end{array}$ \\
\hline-0.40 & 2.8368 & $\begin{array}{r}-29.4002 \\
\quad(0.0297)\end{array}$ & $\begin{array}{c}0.9319 \\
(0.1083)\end{array}$ & 1.4184 & $\begin{array}{c}1.4308 \\
(0.0593)\end{array}$ \\
\hline-0.45 & 3.1990 & $\begin{array}{r}-37.5009 \\
(0.0273)\end{array}$ & $\begin{array}{c}4.3151 \\
(0.2665)\end{array}$ & 1.5995 & $\begin{array}{c}1.6121 \\
(0.1037)\end{array}$ \\
\hline
\end{tabular}


Table VI-Continued

\begin{tabular}{|c|c|c|c|c|c|}
\hline$\rho_{1}$ & $\sigma \times 100$ & $\begin{array}{c}\text { Percentage } \\
\text { Bias }(\hat{s}) \\
(\mathrm{SE})\end{array}$ & $\begin{array}{l}\text { Percentage } \\
\text { Bias }(\hat{\sigma}) \\
\quad(\mathrm{SE})\end{array}$ & $\sqrt{\Delta}$ & $\begin{array}{c}\sqrt{E[\hat{\Delta}]} \\
(\mathrm{SE})\end{array}$ \\
\hline \multicolumn{6}{|c|}{ Panel D. Sample Size $=1,000$} \\
\hline-0.10 & 2.1126 & $\begin{array}{c}-5.3239 \\
(0.0312)\end{array}$ & $\begin{array}{c}0.0578 \\
(0.0433)\end{array}$ & 1.0563 & $\begin{array}{c}1.0569 \\
(0.0249)\end{array}$ \\
\hline-0.20 & 2.2601 & $\begin{array}{r}-11.5035 \\
(0.0293)\end{array}$ & $\begin{array}{c}0.1033 \\
(0.0483)\end{array}$ & 1.1301 & $\begin{array}{c}1.1313 \\
(0.0280)\end{array}$ \\
\hline-0.30 & 2.4716 & $\begin{array}{r}-19.0636 \\
(0.0275)\end{array}$ & $\begin{array}{c}0.1800 \\
(0.0572)\end{array}$ & 1.2358 & $\begin{array}{c}1.2381 \\
(0.0348)\end{array}$ \\
\hline-0.40 & 2.8368 & $\begin{array}{r}-29.4680 \\
(0.0258)\end{array}$ & $\begin{array}{c}0.5782 \\
(0.0885)\end{array}$ & 1.4184 & $\begin{array}{c}1.4271 \\
(0.0535)\end{array}$ \\
\hline-0.45 & 3.1990 & $\begin{array}{r}-37.4445 \\
(0.0236)\end{array}$ & $\begin{array}{c}3.0434 \\
(0.2170)\end{array}$ & 1.5995 & $\begin{array}{c}1.6191 \\
(0.0963)\end{array}$ \\
\hline \multicolumn{6}{|c|}{ Panel E. Sample Size $=1,250$} \\
\hline-0.10 & 2.1126 & $\begin{array}{r}-5.3159 \\
(0.0276)\end{array}$ & $\begin{array}{c}0.0682 \\
(0.0381)\end{array}$ & 1.0563 & $\begin{array}{c}1.0569 \\
(0.0236)\end{array}$ \\
\hline-0.20 & 2.2601 & $\begin{array}{r}-11.5280 \\
(0.0267)\end{array}$ & $\begin{array}{c}0.0517 \\
(0.0434)\end{array}$ & 1.1301 & $\begin{array}{c}1.1310 \\
(0.0264)\end{array}$ \\
\hline-0.30 & 2.4716 & $\begin{array}{r}-19.0691 \\
(0.0256)\end{array}$ & $\begin{array}{c}0.0846 \\
(0.0527)\end{array}$ & 1.2358 & $\begin{array}{c}1.2369 \\
(0.0331)\end{array}$ \\
\hline-0.40 & 2.8368 & $\begin{array}{r}-29.5306 \\
(0.0229)\end{array}$ & $\begin{array}{c}0.3844 \\
(0.0796)\end{array}$ & 1.4184 & $\begin{array}{c}1.4254 \\
(0.0506)\end{array}$ \\
\hline-0.45 & 3.1990 & $\begin{array}{r}-37.4503 \\
(0.0211)\end{array}$ & $\begin{array}{c}2.1408 \\
(0.1700)\end{array}$ & 1.5995 & $\begin{array}{c}1.6206 \\
(0.0854)\end{array}$ \\
\hline
\end{tabular}

underlying stock price process other than what can be inferred from the data, such as the true value of $\sigma .^{28}$ In other words, market option prices imply the true value of $\sigma$ only if some investors actually know $\sigma$. Market prices cannot reveal something that nobody knows. ${ }^{29}$

Even in the case where some investors may have better information about $\sigma$, other investors can rely completely on the implied volatility from market prices only if the prices are informationally efficient (see footnote 29). If there is noise in market option prices due to market imperfections, e.g., frictions,

${ }^{28}$ It should be emphasized that our analysis of the impact of predictability on option prices does not hinge on the particular pricing model we use. In particular, we focus on the Black-Scholes formula solely because of its simplicity. The fact that the Black-Scholes formula may not be correct in its exact form under the maintained assumptions of our analysis - that only discretely sampled data is observed and investors may not be able to trade continuously-affects only the numerical values of our examples, and not the substance of our conclusions.

${ }^{29}$ Of course, one can construct situations where prices can reveal information that no single investor has (see Grossman (1976)), but this is achievable only in the extreme case where prices can efficiently aggregate all the information in the market, i.e., full informational efficiency. For both theoretical and practical reasons, this case is of little interest in modeling financial markets (see Grossman and Stiglitz (1980)). 
deviations from perfect competition, etc., less informed investors will have to rely on their own information as well in making inferences about $\sigma$, such as discretely sampled stock prices (see Figlewski (1989) and Grossman and Stiglitz (1980)).

\section{Conclusion}

The fact that asset return predictability has nontrivial implications for option prices provides a link between two seemingly disparate strands of the asset pricing literature: linear multifactor models of time-varying expected asset returns and arbitrage-based models of derivative asset prices. ${ }^{30}$ Heuristically, when predictability is well defined, i.e., when the asset return's conditional mean does not depend upon past prices or returns, and when the conditional expectation of the prediction error is zero, then increases in predictability generally decrease option prices when the unconditional variance of asset returns is fixed. In such cases, an increase in predictability is equivalent to a reduction in the asset's residual uncertainty or predictionerror variance, and since option prices are monotonically increasing in the volatility of this residual uncertainty in the Black-Scholes case where the diffusion coefficient $\sigma$ is constant, option prices decline as predictability increases.

This has an intriguing implication for the evolution of option premia through time: as we are better able to model the time variation in the expected returns of an asset, option premia on that asset should fall, ceteris paribus. Alternatively, the fact that option premia are positive may imply an upper bound on the predictability of the underlying asset's returns, which may partly address Roll's (1988) lament that $R^{2} \mathrm{~s}$ in financial applications are disappointingly low. We hope to explore these implications in future research.

For alternatives to the Black-Scholes case, such as those with stochastic volatility or jump components, predictability also affects option prices nontrivially, but in considerably more complex ways. To capture such effects, each of our drift specifications can be paired with a particular specification for the diffusion coefficient. While closed-form adjustments for predictability may not always exist in these more general cases, maximum likelihood estimation is almost always feasible for our linear drift specifications.

Despite the fact that the drift of a diffusion process plays virtually no role in deriving theoretical pricing formulas for derivative assets, its importance cannot be overemphasized in the implementation of these formulas. The practical value of arbitrage-based models of derivative prices rests heavily on the existence of an empirically plausible and stable model of the true datagenerating process for the underlying asset's price. Although changing specifications for the drift does not influence the derivative pricing formula, it does

\footnotetext{
${ }^{30}$ At least four other articles have hinted at such a link: Dybvig and Ingersoll (1982), Grundy (1991), Lo (1987), and Sternberg and Ying (1992).
} 
influence both the theoretical value and empirical estimate of the parameter(s) on which the formula depends.

Moreover, although our approach begins by first specifying the true distribution of the data-generating process (which includes the modeling of predictability) and then deriving its implications for the risk-neutral distribution, it is also possible to do the reverse. Taking the properties of the risk-neutral distribution implicit in derivative asset prices as a starting point, it is possible to infer the properties of the true data-generating process (see Grundy (1991), for example). The fact that the risk neutral distribution and the true distribution of the data-generating process are linked makes both directions of inference possible and empirically relevant. ${ }^{31}$

\section{Appendix}

\section{A. The Trending $O-U$ Process}

Under the trending O-U specification (equation (6)) for the detrended price process $q(t)$, the unconditional moments and co-moments of continuously compounded $\tau$-period returns $r_{\tau}(t) \equiv p(t)-p(t-\tau)=\mu \tau+q(t)-q(t-\tau)$ follow readily from the explicit solution in equation (9). ${ }^{32}$

$$
\begin{aligned}
\mathrm{E}\left[r_{\tau}(t)\right] & =\mu \tau \\
\operatorname{Var}\left[r_{\tau}(t)\right] & =\frac{\sigma^{2}}{\gamma}\left[1-e^{-\gamma \tau}\right] \\
\operatorname{Cov}\left[r_{\tau}\left(t_{1}\right), r_{\tau}\left(t_{2}\right)\right] & =-\frac{\sigma^{2}}{2 \gamma} e^{-\gamma\left(t_{2}-t_{1}-\tau\right)}\left[1-e^{-\gamma \tau}\right]^{2}, \quad t_{1}+\tau \leq t_{2} \\
\operatorname{Corr}\left[r_{\tau}(t), r_{\tau}(t+\tau)\right] & \equiv \rho_{\tau}(1)=-\frac{1}{2}\left[1-e^{-\gamma \tau}\right] .
\end{aligned}
$$

${ }^{31}$ Which approach to take, ours or Grundy's, may depend on which market is considered more "efficient" in conveying information: the market for an underlying asset or the market for derivatives of that asset. For example, some have argued that options markets are often more liquid and "informed" than their corresponding spot markets. In such cases, it may well be easier to use option prices to infer predictability in the underlying asset's returns than to study the asset's returns directly. However, since the many recent studies of predictability focus exclusively on the behavior of the underlying asset's data-generating process, we take the true distribution of the data-generating process as our starting point.

${ }^{32}$ Since we have conditioned on $q(0)=q_{0}$ in defining the detrended log-price process, we must be more precise about what we mean by an "unconditional" moment. If $q_{0}$ is assumed to be stochastic and drawn from its stationary distribution, then an unconditional moment of a function of $q(t)$ may be defined as the expectation of the corresponding conditional moment (conditional upon $q_{0}$ ), where the expectation is taken with respect to the stationary distribution of $q_{0}$. Alternatively, if $q(t)$ is stationary, as it is in equation (8), the unconditional moment may be defined as the limit of the corresponding conditional moment as $t$ increases without bound. We shall adopt this definition of an unconditional moment throughout the remainder of the article. In deriving the unconditional moments of $\tau$-period returns, we have assumed that $\gamma>0$. In the special case that $\gamma=0$, the moments can be obtained by simply taking the limit $\gamma \rightarrow 0$ in equations (A11) to (A14). 
Since equation (6) is a Gaussian process, the moments (A1) to (A3) completely characterize the finite-dimensional distributions of $r_{\tau}(t)$.

\section{B. The Bivariate Trending $O-U$ Process}

From equations (29) and (30) we can readily derive the properties of the asset return series that $(q, X)$ generates. To do this, observe that when $\gamma>0$ and $\delta>0$, both $q(t)$ and $X(t)$ are stationary and their first two unconditional moments are:

$$
\begin{aligned}
\mathrm{E}[q(t)] & =\mathrm{E}[X(t)]=0 \\
\operatorname{Var}[q(t)] & =\frac{\sigma^{2}}{2 \gamma}+\frac{\lambda^{2} \sigma_{x}^{2}}{2 \gamma \delta(\gamma+\delta)}+\frac{\lambda \kappa \sigma \sigma_{x}}{\gamma(\gamma+\delta)} \\
\operatorname{Var}[X(t)] & =\frac{\sigma_{x}^{2}}{2 \delta} \\
\operatorname{Cov}[q(t), X(t)] & =\frac{1}{\gamma+\delta}\left(\kappa \sigma \sigma_{x}+\frac{\lambda \sigma_{x}^{2}}{2 \delta}\right) .
\end{aligned}
$$

The unconditional moments of continuously compounded $\tau$-period returns then follow from equations (29) and (30): ${ }^{33}$

$$
\begin{aligned}
\mathrm{E}\left[r_{\tau}(t)\right] & =\mu \tau \\
\operatorname{Var}\left[r_{\tau}(t)\right] & =2 \operatorname{Var}[q(t)]\left[\left(1-e^{-\gamma \tau}\right)-\frac{\lambda}{\gamma-\delta} \beta_{q x}\left(e^{-\delta \tau}-e^{-\gamma \tau}\right)\right]
\end{aligned}
$$

$$
\begin{aligned}
\operatorname{Cov}\left[r_{\tau}(t+\tau), r_{\tau}(t)\right]= & -\operatorname{Var}[q(t)] \\
& \times\left[\frac{\lambda}{\gamma-\delta} \beta_{q x}\left[\left(1-e^{-\delta \tau}\right)^{2}-\left(1-e^{-\gamma \tau}\right)^{2}\right]\right. \\
& \left.+\left(1-e^{-\gamma \tau}\right)^{2}\right] \\
\rho_{\tau}(1)= & -\frac{\left(1-e^{-\gamma \tau}\right)^{2}+\frac{\lambda}{\gamma-\delta} \beta_{q x}\left[\left(1-e^{-\delta \tau}\right)^{2}-\left(1-e^{-\gamma \tau}\right)^{2}\right]}{2\left[\left(1-e^{-\gamma \tau}\right)-\frac{\lambda}{\gamma-\delta} \beta_{q x}\left(e^{-\delta \tau}-e^{-\gamma \tau}\right)\right]}
\end{aligned}
$$

${ }^{33}$ If $\gamma=0$ or $\delta=0$, the unconditional moments of $q(t)$ and $X(t)$ may not exist. However, the unconditional moments of returns are always well defined and may be obtained by taking the appropriate limits in the following results. 
where $\beta_{q x} \equiv \operatorname{Cov}[q(t), X(t)] / \operatorname{Var}[q(t)]$ and $\rho_{\tau}(1)$ is the first-order autocorrelation function of $\tau$-period returns.

A closely related quantity that may help to develop further intuition for the bivariate trending O-U process is the general autocorrelation function $\rho_{\tau}(k)$, defined as the correlation between two $\tau$-period continuously compounded returns that are $(k-1) \tau$ periods apart, i.e.,

$$
\rho_{\tau}(k) \equiv \frac{\operatorname{Cov}\left[r_{\tau}(t+k \tau), r_{\tau}(t)\right]}{\operatorname{Var}\left[r_{\tau}(t)\right]} .
$$

Observe that the first-order autocorrelation function $\rho_{\tau}(1)$, defined in equation (A4), is indeed a special case of this more general definition. In the case of the bivariate trending $\mathrm{O}-\mathrm{U}$ process, the autocorrelation function is given by:

$$
\rho_{\tau}(k)=e^{-(k-1) \gamma \tau} \rho_{\tau}(1)-\frac{\lambda}{\gamma-\delta}\left[e^{-(k-1) \delta \tau}-e^{-(k-1) \gamma \tau}\right] \theta(\tau)
$$

where

$$
\theta(\tau) \equiv \frac{\beta_{q x}\left(1-e^{-\delta \tau}\right)^{2}}{2\left[\left(1-e^{-\gamma \tau}\right)-\frac{\lambda}{\gamma-\delta} \beta_{q x}\left(e^{-\delta \tau}-e^{-\gamma \tau}\right)\right]} .
$$

To derive equations (47) and (48), observe that from equations (26) and (27) we have:

$$
\begin{aligned}
q\left(t_{k}\right)= & q\left(t_{k-1}\right) e^{-\gamma \tau}+\frac{\lambda}{\gamma-\delta}\left(e^{-\delta \tau}-e^{-\gamma \tau}\right) X\left(t_{k}\right) \\
& +\int_{t_{k-1}}^{t_{k}} e^{-\gamma\left(t_{k}-s\right)} \sigma d W_{q}(s)+\frac{\lambda}{\gamma-\delta} \int_{t_{k-1}}^{t_{k}}\left[e^{-\delta\left(t_{k}-s\right)}-e^{-\gamma\left(t_{k}-s\right)}\right] \sigma_{x} d W_{x}(s)
\end{aligned}
$$

$$
X\left(t_{k}\right)=e^{-\delta \tau} X\left(t_{k-1}\right)+\int_{t_{k-1}}^{t_{k}} e^{-\delta\left(t_{k}-s\right)} \sigma_{x} d W_{x}(s) .
$$

Define $\alpha_{q} \equiv e^{-\gamma \tau}, \alpha_{x} \equiv e^{-\delta \tau}, \phi=\lambda\left(\alpha_{x}-\alpha_{q}\right) /(\gamma-\delta)$, and

$$
\begin{aligned}
& \epsilon_{q, k}=\int_{t_{k-1}}^{t_{k}} e^{-\gamma\left(t_{k}-s\right)} \sigma d W_{q}(s)+\frac{\lambda}{\gamma-\delta} \int_{t_{k-1}}^{t_{k}}\left[e^{-\delta\left(t_{k}-s\right)}-e^{-\gamma\left(t_{k}-s\right)}\right] \sigma_{x} d W_{x}(s) \\
& \epsilon_{x, k}=\int_{t_{k-1}}^{t_{k}} e^{-\delta\left(t_{k}-s\right)} \sigma_{x} d W_{x}(s) .
\end{aligned}
$$

We then have:

$$
\begin{aligned}
q_{k} & =\alpha_{q} q_{k-1}+\phi X_{k-1}+\epsilon_{q, k} \\
X_{k} & =\alpha_{x} X_{k-1}+\epsilon_{x, k} .
\end{aligned}
$$


Clearly, $\epsilon_{q, k}$ and $\epsilon_{x, k}$ are independently and identically distributed over time and jointly normally distributed. Furthermore,

$$
\begin{aligned}
& s_{q, \tau}^{2} \equiv \operatorname{Var}\left[\epsilon_{q, k}\right]= \frac{\sigma^{2}}{2 \gamma}\left(1-\alpha_{q}^{2}\right)+\frac{2 \lambda \kappa \sigma_{x} \sigma}{\gamma-\delta}\left[\frac{1-\alpha_{q} \alpha_{x}}{\gamma+\delta}-\frac{1-\alpha_{q}^{2}}{2 \gamma}\right] \\
&+\frac{\lambda^{2} \sigma_{x}^{2}}{(\gamma-\delta)^{2}}\left[\frac{1-\alpha_{q}^{2}}{2 \gamma}+\frac{1-\alpha_{x}^{2}}{2 \delta}-\frac{2\left(1-\alpha_{q} \alpha_{x}\right)}{\gamma+\delta}\right] \\
& s_{x, \tau}^{2} \equiv \operatorname{Var}\left[\epsilon_{x, k}\right]= \frac{\sigma_{x}^{2}}{2 \delta}\left(1-\alpha_{x}^{2}\right) \\
& s_{q x, \tau} \equiv \operatorname{Cov}\left[\epsilon_{q, k}, \epsilon_{x, k}\right]=\frac{\kappa \sigma_{x} \sigma}{\gamma+\delta}\left(1-\alpha_{q} \alpha_{x}\right)+\frac{\lambda \sigma_{x}^{2}}{\gamma-\delta}\left[\frac{1-\alpha_{x}^{2}}{2 \delta}-\frac{1-\alpha_{q} \alpha_{x}}{\gamma+\delta}\right]
\end{aligned}
$$

There is a one-to-one mapping between the parameters of the discretely sampled system, $\alpha_{q}, \alpha_{x}, \phi, s_{q, \tau}^{2}, s_{x, \tau}^{2}$, and $s_{q x, \tau}$, and the parameters of the underlying continuous-time process, $\gamma, \delta, \lambda, \sigma, \sigma_{x}$, and $\kappa$. Specifically, normalize the time units so that $\tau=1$ and observe that:

$$
\gamma=-\log \alpha_{q}, \quad \delta=-\log \alpha_{x}, \quad \lambda=\frac{\phi\left(\log \alpha_{q}-\log \alpha_{x}\right)}{\alpha_{q}-\alpha_{x}} .
$$

Substituting equation (A24) into equations (A21) to (A23) then yields three equations that are linear in $\sigma^{2}, \sigma_{x}^{2}$, and $\kappa \sigma \sigma_{x}$, hence the remaining three continuous-time parameters may be easily recovered from these equations.

Since $\left(p_{k}, X_{k}\right)$ follows a bivariate $\operatorname{AR}(1)$ process, a closed-form expression for the likelihood function of $p_{k}$ may be obtained that can be used in the maximum likelihood estimation (e.g., Jazwinski (1970)).

\section{The Multivariate Trending $O-U$ Process}

The multivariate trending O-U process $\left(q(t), \mathbf{X}^{\prime}(t)\right)$ is defined by the following Itô integrals:

$$
\begin{aligned}
q(t)= & e^{-\gamma t} q_{0}+\Lambda(\gamma \mathbf{I}-\Delta)^{-1}\left[e^{-\Delta t}-e^{-\gamma \mathbf{I} t}\right] \mathbf{X}_{0}+\int_{0}^{t} e^{-\gamma(t-s)} \sigma d W_{q}(s) \\
& -\Lambda(\gamma \mathbf{I}-\Delta)^{-1} \int_{0}^{t}\left[e^{-\gamma \mathbf{I}(t-s)}-e^{-\Delta(t-s)}\right] \mathbf{B}_{x} d \mathbf{W}_{x}(s) \\
\mathbf{X}(t)= & e^{-\Delta t} \mathbf{X}_{0}+\int_{0}^{t} e^{-\Delta(t-s)} \mathbf{B}_{x} d \mathbf{W}_{x}(s)
\end{aligned}
$$

where $\mathbf{I}$ is the $(m \times m)$ identity matrix. When $\gamma$ and the real parts of all of the eigenvalues of $\Delta$ are strictly positive, $\left(q(t), \mathbf{X}^{\prime}(t)\right)$ is stationary. The unconditional moments of $q(t)$ and $\mathbf{X}(t)$ may be readily obtained from equations (A25) and (A26). 
Since $\mathbf{W}_{x}(t)$ is a $k$-dimensional standard Wiener process $\mathrm{E}\left[d \mathbf{W}_{x} d \mathbf{W}_{x}^{\prime}\right]=\mathbf{I} d t$ where $\mathbf{I}$ is the identity matrix of order $k .^{34}$ Let $\sigma d W_{q} \mathbf{B}_{x} d \mathbf{W}_{x}=\mathbf{K} d t$ where $\mathbf{K}$ is a $(k \times 1)$ vector. For notational convenience, define $\Sigma=\left\{\sigma_{i j}\right\} \equiv \mathbf{B}_{x} \mathbf{B}_{x}^{\prime}$, $\Omega_{\tau}=\left\{\omega_{i j}(\tau)\right\}$ where $\omega_{i j}(\tau) \equiv \sigma_{i j}\left[1-e^{-\left(\delta_{i}+\delta_{j}\right) \tau}\right] /\left(\delta_{i}+\delta_{j}\right)$ and $\Xi_{\tau}=\left\{\xi_{i j}(\tau)\right\}$ where $\xi_{i j}(\tau) \equiv \sigma_{i j}\left[1-e^{-\left(\gamma+\delta_{j}\right) \tau}\right] /\left(\gamma+\delta_{j}\right)$.

Then we have:

$$
\begin{aligned}
\mathrm{E}[q(t)]= & \mathrm{E}[\mathbf{X}(t)]=0 \\
\operatorname{Var}[q(t)]= & \frac{\sigma^{2}}{2 \gamma}+\Lambda(\gamma \mathbf{I}-\Delta)^{-1}\left(\frac{1}{2 \gamma} \Sigma-\Xi_{\infty}-\Xi_{\infty}^{\prime}+\Omega_{\infty}\right) \\
& \times(\gamma \mathbf{I}-\Delta)^{-1} \Lambda^{\prime} \\
& +\Lambda(\gamma \mathbf{I}-\Delta)^{-1}\left[2(\gamma \mathbf{I}+\Delta)^{-1}-\frac{1}{\gamma} \mathbf{I}\right] \mathbf{K} \\
\operatorname{Var}[\mathbf{X}(t)]= & \Omega_{\infty} \\
\operatorname{Cov}[q(t), \mathbf{X}(t)]= & \left(\Xi_{\infty}-\Omega_{\infty}\right)(\gamma \mathbf{I}-\Delta)^{-1} \Lambda^{\prime}+(\gamma \mathbf{I}+\Delta)^{-1} \mathbf{K}
\end{aligned}
$$

$\operatorname{Cov}[q(t), q(t+\tau)]=e^{-\gamma \tau} \operatorname{Var}[q(t)]$

$$
+\Lambda(\gamma \mathbf{I}-\Delta)^{-1}\left(e^{-\Delta \tau}-e^{-\gamma \mathbf{I} \tau}\right) \operatorname{Cov}[q(t), \mathbf{X}(t)] .
$$

From these expressions, the moments of $r_{\tau}(t)$ follow directly:

$$
\begin{aligned}
\mathrm{E}\left[r_{\tau}(t)\right]= & \mu \tau \\
\operatorname{Var}\left[r_{\tau}(t)\right]= & 2 \operatorname{Var}[q(t)]\left[\left(1-e^{-\gamma \tau}\right)-\Lambda(\gamma \mathbf{I}-\Delta)^{-1}\right. \\
& \left.\times\left(e^{-\Delta \tau}-e^{-\gamma \mathbf{I} \tau}\right) \mathbf{b}\right] \\
\operatorname{Cov}\left[r_{\tau}(t+\tau), r_{\tau}(t)\right]= & -\operatorname{Var}[q(t)]\left\{\left(1-e^{-\gamma \tau}\right)^{2}\right. \\
& \left.+\Lambda(\gamma \mathbf{I}-\Delta)^{-1}\left[\left(\mathbf{I}-e^{-\Delta \tau}\right)^{2}-\left(\mathbf{I}-e^{-\gamma \mathbf{I} \tau}\right)^{2}\right] \mathbf{b}\right\}
\end{aligned}
$$

where $\mathbf{b} \equiv \operatorname{Cov}[q(t), \mathbf{X}(t)] / \operatorname{Var}[q(t)]$. The return autocorrelation function may then be obtained from these moments.

It is straightforward to derive the discrete-time representation of the system $\left(q(t), \mathbf{X}^{\prime}(t)\right)$ :

$$
\begin{aligned}
q_{k} & =\alpha_{q} q_{k-1}+\Phi \mathbf{X}_{k-1}+\epsilon_{q, k} \\
\mathbf{X}_{k} & =\mathbf{A}_{x} \mathbf{X}_{k-1}+\epsilon_{x, k}
\end{aligned}
$$

\footnotetext{
${ }^{34}$ There is no loss of generality by assuming that $\mathbf{W}_{x}(t)$ has independent components since components of $\mathbf{X}(t)$ can have arbitrary covariance structure through $\mathbf{B}_{x}$.
} 
where $q_{k} \equiv q\left(t_{k}\right), \quad \mathbf{X}_{k} \equiv \mathbf{X}\left(t_{k}\right), \quad \tau \equiv t_{k}-t_{k-1}, \quad \alpha_{q} \equiv e^{-\gamma \tau}, \quad \mathbf{A}_{x} \equiv e^{-\Delta \tau}, \quad \Phi \equiv$ $\Lambda(\gamma \mathbf{I}-\Delta)^{-1}\left(\mathbf{A}_{x}-\alpha_{q} \mathbf{I}\right)$

and

$$
\begin{aligned}
\epsilon_{q, k} \equiv & \int_{t_{k-1}}^{t_{k}} e^{-\gamma\left(t_{k}-s\right)} \sigma d W_{q}(s) \\
& -\Lambda(\gamma \mathbf{I}-\Delta)^{-1} \int_{t_{k-1}}^{t_{k}}\left[e^{-\gamma \mathbf{I}\left(t_{k}-s\right)}-e^{-\Delta\left(t_{k}-s\right)}\right] \mathbf{B}_{x} d \mathbf{W}_{x}(s) \\
\boldsymbol{\epsilon}_{x, k} \equiv & \int_{t_{k-1}}^{t_{k}} e^{-\Delta\left(t_{k}-s\right)} \mathbf{B}_{x} d \mathbf{W}_{x}(s) .
\end{aligned}
$$

It is easy to show that

$$
\begin{aligned}
s_{q, \tau}^{2} \equiv \operatorname{Var}\left[\epsilon_{q, k}\right]= & \frac{1-\alpha_{q}^{2}}{2 \gamma} \sigma^{2}+\Lambda(\gamma \mathbf{I}-\Delta)^{-1} \\
& \times\left(\frac{1-\alpha_{q}^{2}}{2 \gamma} \Sigma-\Xi_{\tau}-\Xi_{\tau}^{\prime}+\Omega_{\tau}\right)(\gamma \mathbf{I}-\Delta)^{-1} \Lambda^{\prime} \\
& +\Lambda(\gamma \mathbf{I}-\Delta)^{-1}\left\{2(\gamma \mathbf{I}+\Delta)^{-1}\left[1-e^{-(\gamma \mathbf{I}+\Delta) \tau}\right]-\frac{1-\alpha_{q}^{2}}{\gamma} \mathbf{I}\right\} \mathbf{K} \\
s_{x, \tau}^{2} \equiv & \operatorname{Var}\left[\epsilon_{x, k}\right]=\Omega_{\tau} \\
s_{q x, \tau} \equiv & \operatorname{Cov}\left[\epsilon_{q, k}, \epsilon_{x, k}\right] \\
= & \left(\Omega_{\tau}-\Xi_{\tau}\right)(\gamma \mathbf{I}-\Delta)^{-1} \Lambda^{\prime}+(\gamma \mathbf{I}+\Delta)^{-1}\left[1-e^{-(\gamma \mathbf{I}+\Delta) \tau}\right] \mathbf{K}
\end{aligned}
$$

Similar to the bivariate case, the mapping between the parameters of the discrete-time representation, $\alpha_{q}, \mathbf{A}_{x}, \Phi, s_{q, \tau}^{2}, s_{x, \tau}^{2}$, and $s_{q x, \tau}$, and the parameters of the underlying continuous-time process, $\gamma, \Delta, \Lambda, \sigma^{2}, \Sigma$, and $\mathbf{K}$ is one to one. ${ }^{35}$ Let $\tau=1$, we have

$$
\gamma=-\log \alpha_{q}, \quad \delta_{i}=-\log \alpha_{i}, \quad \Lambda=\Phi\left(\mathbf{A}_{x}-\alpha_{q} \mathbf{I}\right)^{-1}(\gamma \mathbf{I}-\Delta)
$$

where $\alpha_{i}=\left\{\mathbf{A}_{x}\right\}_{i i}$ (note that $\mathbf{A}_{x}$ is diagonal). From $s_{x, \tau}^{2}$, we can solve for $\Omega_{\tau}$, $\Sigma$ and $\Xi_{\tau}$ :

$$
\Omega_{\tau}=s_{x, \tau}^{2}, \quad \sigma_{i j}=\frac{\left(\delta_{i}+\delta_{j}\right) \omega_{i j}(\tau)}{1-\alpha_{i} \alpha_{j}}, \quad \xi_{i j}=\frac{\sigma_{i j}\left(1-\alpha_{i} \alpha_{j}\right)}{\gamma+\delta_{j}} .
$$

We can then solve for $\mathbf{K}$ given $s_{q x, \tau}$ :

$$
\mathbf{K}=\left[1-e^{-(\gamma \mathbf{I}+\Delta)}\right]^{-1}(\gamma \mathbf{I}+\Delta)\left[s_{q x, \tau}-\left(\Omega_{\tau}-\Xi_{\tau}\right)(\gamma \mathbf{I}-\Delta)^{-1} \Lambda^{\prime}\right] .
$$

From the definition of $s_{q, \tau}^{2}$, we can further solve for $\sigma^{2}$.

${ }^{35}$ Note that $\mathbf{B}_{x}$ is simply the Cholesky decomposition of $\Sigma$. 


\section{REFERENCES}

Abel, A., 1992, Exact solutions for expected rates of return under Markov regime switching: Implications for the equity premium puzzle, National Bureau of Economic Research (NBER) Working paper No. 4110.

Barry, C., D. French, and R. Rao, 1991, Estimation risk and adaptive behavior in the pricing of options, Financial Review 26, 15-30.

Bekaert, G. and R. Hodrick, 1992, Characterizing predictable components in excess returns on equity and foreign exchange markets, Journal of Finance 47, 467-509.

Bessembinder, H. and K. Chan, 1992, Time-varying risk premia and forecastable returns in futures markets, Journal of Financial Economics 32, 169-194.

Black, F. and M. Scholes, 1972, The valuation of option contracts and a test of market efficiency, Journal of Finance 27, 399-418.

1973, The pricing of options and corporate liabilities, Journal of Political Economy 81, 637-659.

Boyle, P. and D. Emanuel, 1980, Discretely adjusted option hedges, Journal of Financial Economies 8, 259-282.

Breen, W., L. Glosten, and R. Jagannathan, 1989, Economic significance of predictable variations in stock index returns, Journal of Finance 44, 1177-1189.

Campbell, J. and J. Ammer, 1993, What moves the stock and bond markets? A variance decomposition for long-term asset returns, Journal of Finance 48, 3-37.

Campbell, J. and Y. Hamao, 1992, Predictable stock returns in the United States and Japan: A study of long-term capital market integration, Journal of Finance 47, 43-70.

Campbell, J. and P. Perron, 1991, Pitfalls and opportunities: What macroeconomists should know about unit roots, in O. Blanchard and S. Fischer, Eds.: NBER Macroeconomics Annual (MIT Press: Cambridge), pp. 141-201.

Cecchetti, S., P. Lam, and N. Mark, 1993. The equity premium and the risk free rate: matching the moments, Journal of Monetary Economy 31, 21-45.

Chan, K., 1992, A further analysis of the lead-lag relationship between the cash market and stock index futures market, Review of Financial Studies 5, 123-152.

Chen, N., 1991, Financial investment opportunities and the macroeconomy, Journal of Finance $46,529-554$.

- , R. Roll, and S. Ross, 1986, Economic forces and the stock market, Journal of Business $59,383-403$.

Chopra, N., J. Lakonishok, and J. Ritter, 1992, Measuring abnormal performance: Do stocks overreact? Journal of Financial Economics 31, 235-268.

Cox, J. and S. Ross, 1976, The valuation of options for alternative stochastic processes, Journal of Financial Economics 3, 145-166.

DeBondt, W. and R. Thaler, 1985, Does the stock market overreact? Journal of Finance 40, 793-805.

Doob, J., 1953, Stochastic Processes (John Wiley \& Sons, New York).

Dybvig, P. and J. Ingersoll, 1982, Mean-variance theory in complete markets, Journal of Business 55, 233-251.

Engle, R., D. Lilien, and R. Robbins, 1987, Estimating time varying risk premia in the term structure: The ARCH-M model, Econometrica 55, 391-407.

Fama, E. and K. French, 1988a, Permanent and temporary components of stock prices, Journal of Political Economy 96, 246-273.

, 1988b, Dividend yields and expected stock returns, Journal of Financial Economics 22, 3-26.

,- 1990 , Business conditions and expected returns on stocks and bonds, Journal of Financial Economics 25, 23-49.

Ferson, W., 1989, Changes in expected security returns, risk, and the level of interest rates, Journal of Finance 44, 1191-1217.

,- 1990 , Are the latent variables in time-varying expected returns compensation for consumption risk? Journal of Finance 45, 397-430.

- S. Foerster, and D. Keim, 1993, General tests of latent variable models and mean-variance spanning, Journal of Finance 48, 131-156. 
Ferson, W. and C. Harvey, 1991a. Sources of predictability in portfolio returns, Financial Analysts Journal May-June, 49-56.

$-1991 b$, The variation of economic risk premiums, Journal of Political Economy 99, $385-415$.

Ferson, W., S. Kandel, and R. Stambaugh, 1987, Tests of asset pricing with time-varying expected risk premiums and market betas, Journal of Finance 42, 201-220.

Figlewski, S., 1989, What does an option pricing model tell us about option prices? Financial Analysts Journal 45, 12-15.

Gibbons, M. and W. Ferson, 1985, Testing asset pricing models with changing expectations and an unobservable market portfolio, Journal of Financial Economics 14, 217-236.

Grossman, S., 1976, On the efficiency of competitive stock markets where traders have diverse information, Journal of Finance 31, 573-585.

- , and J. Stiglitz, 1980, On the impossibility of informationally efficient markets, American Economic Review 70, 393-408.

Grundy, B., 1991, Option prices and the underlying asset's return distribution, Journal of Finance 46, 1045-1069.

Harrison, M. and D. Kreps, 1979, Martingales and multiperiod securities markets, Journal of Economic Theory 20, 381-408.

Harrison, M., R. Pitbladdo, and S. Schaefer, 1984, Continuous price processes in frictionless markets have infinite variation, Journal of Business 57, 353-365.

Harvey, A., 1989a, Forecasting, Structure Time Series Models and the Kalman Filter (Cambridge University Press, Cambridge, U.K.).

Harvey, A., 1989b, Time-Varying conditional covariances in tests of asset pricing models, Journal of Financial Economics 24, 289-317.

Heaton, J. and D. Lucas, 1992, The effects of incomplete insurance markets and trading costs in a consumption-based asset pricing model, Journal of Economic Dynamics and Control 16, 601-620.

- 1994, Evaluating the effects of incomplete markets on risk sharing and asset pricing, Working paper, Sloan School of Management, Massachusetts Institute of Technology.

Huang, C. and A. Lo, 1994, Estimating the drift of a general diffusion process, Working paper Sloan School of Management, Massachusetts Institute of Technology.

Jagannathan, R., 1984, Call options and the risk of underlying securities, Journal of Financial Economics 13, 425-434.

Jazwinski, A., 1970, Stochastic Processes and Filtering Theory. Academic Press, New York.

Jegadeesh, N., 1990, Evidence of predictable behavior of security returns, Journal of Finance 45, 881-898.

Kandel, S. and R. Stambaugh, 1988, Modeling expected stock returns for long and short horizons, Rodney L. White Center Working paper No. 42-88, Wharton School, University of Pennsylvania.

,- 1990 , Expectations and volatility of consumption and asset returns, Review of Financial Studies 3, 207-232.

Keim, D. and R. Stambaugh, 1986, Predicting returns in the stock and bond markets, Journal of Financial Economics 17, 357-390.

King, B., 1966, Market and industry factors in stock price behavior, Journal of Business 39, $139-190$.

Lehmann, B., 1990, Fads, martingales, and market efficiency, Quarterly Journal of Economics $105,1-28$.

Leland, H., 1985, Option pricing and replication with transactions costs, Journal of Finance 40, 1283-1301.

Lo, A., 1986, Statistical tests of contingent claims asset-pricing models: A new methodology, Journal of Financial Economics 17, 143-173.

- 1987, Semiparametric upper bounds for option prices and expected payoffs, Journal of Financial Economics 19, 373-388.

,- 1988 , Maximum likelihood estimation of generalized Itô processes with discretely-sampled data, Econometric Theory 4, 231-247. 
and C. MacKinlay, 1988, Stock market prices do not follow random walks: Evidence from a simple specification test, Review of Financial Studies 1, 41-66.

, 1990, When are contrarian profits due to stock market overreaction? Review of Financial Studies 3, 175-206.

- 1992, Maximizing predictability in the stock and bond markets, Working paper No. 3450-92-EFA, Sloan School of Management, Massachusetts Institute of Technology.

Lütkepohl, H., 1991, Introduction to Multiple Time Series Analysis (Springer-Verlag, Berlin, Germany).

Mehra, R. and E. Prescott, 1985, The equity premium: A puzzle, Journal of Monetary Economy 15, 145-162.

Merton, R., 1973, Rational theory of option pricing, Bell Journal of Economics and Management Science 4, 141-183.

-1 , 1976a, Option pricing when underlying stock returns are discontinuous, Journal of Financial Economics 3, 125-144.

- $1976 \mathrm{~b}$, The impact on option pricing of specification error in the underlying stock price distribution, Journal of Finance 31, 333-350.

Poterba, J. and L. Summers, 1988, Mean reversion in stock returns: Evidence and implications, Journal of Financial Economics 22, 27-60.

Roll, R., 1988, $R^{2}$, Journal of Finance 43, 541-566.

Sims, C., 1984, Martingale-like behavior of prices and interest rates, Discussion paper No. 205, Center of Economic Research, Department of Economics, University of Minnesota.

Sternberg, J. and J. Ying, 1992, Option pricing for assets with serially correlated returns, Working paper, University of Arizona.

Weil, P., 1989, The equity premium puzzle and the risk-free rate puzzle, Journal of Monetary Economy 24, 401-421. 